\title{
Generalized polarizabilities of the nucleon in baryon chiral perturbation theory
}

\author{
Vadim Lensky $^{1,2,3, a}$, Vladimir Pascalutsa ${ }^{1}$, Marc Vanderhaeghen ${ }^{1}$ \\ ${ }^{1}$ Institut für Kernphysik, Cluster of Excellence PRISMA, Johannes Gutenberg Universität Mainz, 55128 Mainz, Germany \\ ${ }^{2}$ Institute for Theoretical and Experimental Physics, Moscow 117218, Russia \\ ${ }^{3}$ National Research Nuclear University MEPhI (Moscow Engineering Physics Institute), Moscow 115409, Russia
}

Received: 9 January 2017 / Accepted: 26 January 2017 / Published online: 21 February 2017

(C) The Author(s) 2017. This article is published with open access at Springerlink.com

\begin{abstract}
The nucleon generalized polarizabilities (GPs), probed in virtual Compton scattering (VCS), describe the spatial distribution of the polarization density in a nucleon. They are accessed experimentally via the process of electronproton bremsstrahlung $(e p \rightarrow e p \gamma)$ at electron-beam facilities, such as MIT-Bates, CEBAF (Jefferson Lab), and MAMI (Mainz). We present the calculation of the nucleon GPs and VCS observables at next-to-leading order in baryon chiral perturbation theory $(\mathrm{B} \chi \mathrm{PT})$, and confront the results with the empirical information. At this order our results are predictions, in the sense that all the parameters are well known from elsewhere. Within the relatively large uncertainties of our calculation we find good agreement with the experimental observations of VCS and the empirical extractions of the GPs. We find large discrepancies with previous chiral calculations - all done in heavy-baryon $\chi \mathrm{PT}(\mathrm{HB} \chi \mathrm{PT})-$ and discuss the differences between $\mathrm{B} \chi \mathrm{PT}$ and $\mathrm{HB} \chi \mathrm{PT}$ responsible for these discrepancies.
\end{abstract}

\section{Contents}

1 Introduction . . . . . . . . . . . . . 1

2 Polarizabilities in Compton processes . . . . . . . 2

3 Chiral perturbation theory of generalized polarizabilities ................ 4

3.1 Further remarks on power counting . . . . . . 5

3.2 In practice . . . . . . . . . . . . . 6

3.3 Error estimate . . . . . . . . . . . . 7

4 Comparison with previous calculations ... . . . 8

4.1 Linear $\sigma$-model . . . . . . . . . . . . . . . . . 8

Electronic supplementary material The online version of this article (doi:10.1140/epjc/s10052-017-4652-9) contains supplementary material, which is available to authorized users.

a e-mail: lensky@itep.ru
4.2 Heavy-baryon expansion . . . . . . . . . 8

4.3 Fixed- $t$ dispersion relations . . . . . . . . . . 10

5 Results for VCS observables . . . . . . . . . . . . 11

6 Concluding remarks . . . . . . . . . . 12

Appendix A: Tensor decomposition of the

VCS amplitude . . . . . . . . . . . . . 13

Appendix B: Invariant amplitudes . . . . . . . . . . 14

$1 . \pi N$ loops . . . . . . . . . . . . . 14

a. Proton . . . . . . . . . . . . . . . 14

b. Neutron . . . . . . . . . . . . . . 15

2. Delta pole . . . . . . . . . . . . 15

References . . . . . . . . . . . . . . 16

\section{Introduction}

Long after the early studies of the electron-proton (ep) bremsstrahlung [1,2], it was realized that this process holds the key to the generalized polarizabilities (GPs) of the nucleon [3]; see Ref. [4] for a review. The GPs extend the concept of static polarizabilities to finite momentum transfer $Q^{2}$, and have an interpretation of the distribution of polarization densities in the nucleon [5]. They naturally arise in virtual Compton scattering (VCS) with the incoming virtual photon of spacelike virtuality $Q^{2}$, and the outgoing real photon of very low frequency; hence we have the $e p$ bremsstrahlung which, in the one-photon-exchange approximation, decomposes into the Bethe-Heitler $(\mathrm{BH})$ process and VCS; cf. Fig. 1. Shown in the figure is also the split of VCS into: A) the Born contribution to VCS, with the intermediate state being the nucleon itself, and B) non-Born contribution to VCS, which at low energies is entirely determined by GPs $[3,6,7]$. The BH and Born VCS contributions are given in terms of the electromagnetic nucleon form factors known from elastic electron scattering. The non-Born VCS amplitude, carrying the information about the "inelastic" structure 

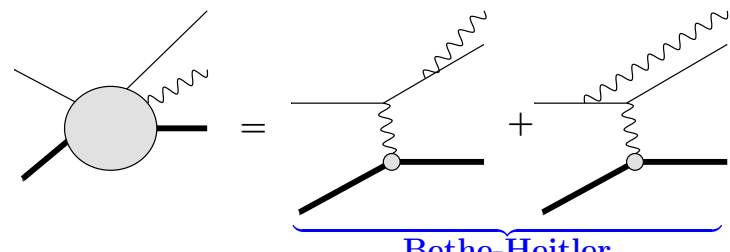

Bethe-Heitler

Fig. 1 Mechanisms contributing to $e p \rightarrow e p \gamma$ in the one-photonexchange approximation: Bethe-Heitler, Born VCS, non-Born VCS. Thick (thin) solid lines denote the proton (the electron), wavy lines

of the nucleon, is the unknown piece that one is trying to access in the $e p$ bremsstrahlung.

Over the past two decades the experimental studies clearly demonstrated the feasibility of an accurate extraction of proton GPs from ep bremsstrahlung [8-14]. This experimental progress has been echoed by theory advances. A number of impressive calculations have been done in heavy-baryon chiral perturbation theory $(\mathrm{HB} \chi \mathrm{PT})$ [15-19], albeit showing a rather poor convergence. A much more empirically viable theory of proton GPs and VCS was developed by Pasquini et al. [20,21] based on fixed- $t$ dispersive relations (DRs) for the VCS amplitudes. Incidentally, this framework is used in many experimental studies to extract the GPs from the VCS observables.

The present work is aiming to advance the chiral effectivefield theoretic approach by applying the manifestly Lorentzinvariant variant of baryon chiral perturbation theory $(\mathrm{B} \chi \mathrm{PT})$ to nucleon VCS and GPs. As many recent calculations demonstrate (see, e.g., [22-31]), B $\chi$ PT shows an improved convergence over the analogous $\mathrm{HB} \chi \mathrm{PT}$ calculations, and, as result, a more "natural" description of the nucleon polarizabilities and Compton scattering processes [32-36]. In this paper, we extend the previous $\mathrm{B} \chi \mathrm{PT}$ calculations of Lensky et al. [35-37], done for nucleon polarizabilities appearing in real and forward doubly virtual Compton scattering (RCS and VVCS, respectively), to the case of GPs and VCS. As in the previous cases, the present calculation is "predictive" in the sense that it has no free parameters to be fixed by the empirical information from Compton processes. And, as in other cases, we find significant improvements in convergence over the analogous $\mathrm{HB} \chi \mathrm{PT}$ results. Arguably, the main improvement is that our postdictions compare well with the experimental data on VCS observables, at least given the significant theoretical uncertainties.

The paper is organized as follows. In Sect. 2, we open with the general remarks concerning the connection between polarizabilities and low-energy Compton scattering processes, and then focus on defining the GPs and the VCS observables. Section 3 contains the details of our B $\chi$ PT calculation, including power counting, diagrams, theory error estimate, and remarks on a number of technical issues which arise in these calculations. Section 4 compares our calcula-

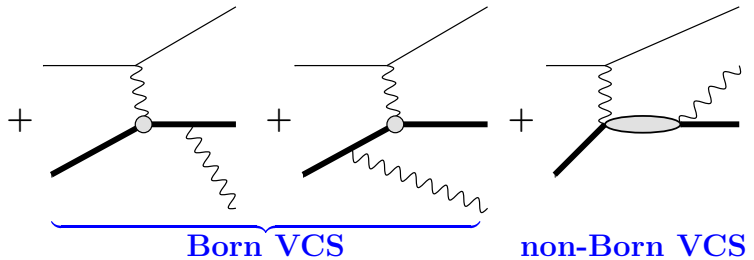

denote photons. Small circles denote the interaction vertex of a proton with a virtual photon, and the ellipse stands for the generic non-Born VCS amplitude

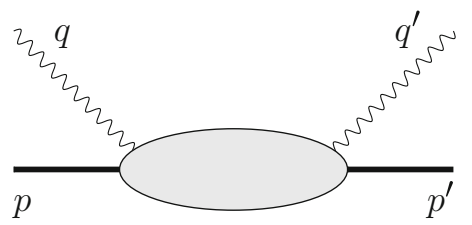

Fig. 2 General Compton scattering process. The notation is as in Fig. 1, with the four-momenta of the corresponding particles denoted in the figure

tion with previous estimates: the linear $\sigma$-model, $\mathrm{HB} \chi \mathrm{PT}$ calculations, and fixed- $t$ dispersive estimates. Section 5 confronts the results with the available experimental data. Section 6 contains the concluding remarks. Appendix A contains expressions for the tensors that are used in the decomposition of the VCS amplitude, whereas Appendix B contains analytic expressions for those combinations of the invariant VCS amplitudes that contribute to the GPs.

\section{Polarizabilities in Compton processes}

Let us start by pointing out that there are two different ways of introducing the momentum-transfer dependence of polarizabilities: one via the forward doubly virtual Compton scattering (VVCS), the other via the single-virtual Compton scattering (VCS). To see the difference, consider a general Compton scattering (CS) process in Fig. 2, described by a number of scalar amplitudes $A_{i}$, functions of Mandelstam invariants

$$
\begin{aligned}
& s=(p+q)^{2}=\left(p^{\prime}+q^{\prime}\right)^{2}, \quad t=\left(q-q^{\prime}\right)^{2}=\left(p^{\prime}-p\right)^{2}, \\
& u=\left(p-q^{\prime}\right)^{2}=\left(p^{\prime}-q\right)^{2} .
\end{aligned}
$$

The latter satisfy the usual kinematical constraint,

$s+u+t=2 M^{2}+q^{2}+q^{\prime 2}$,

with $M$ the nucleon mass and $q^{2}, q^{\prime 2}$ the photon virtualities. The polarizabilities can be equated with the coefficients in the low-energy expansion of the CS amplitudes $A_{i}$. Introducing the invariant energies of the incoming and outgoing photon: $v=p \cdot q / M$ and $v^{\prime}=p \cdot q^{\prime} / M$, the low-energy expansion requires at least one of them to be small. Note that the kinematical constraint can be written as $t=-2 M\left(v-v^{\prime}\right)$, hence by energy conservation $t \leq 0$. It is convenient to introduce the kinematic invariant, 
$\xi=\frac{s-u}{4 M}$,

which is odd under the photon crossing, $\xi \rightarrow-\xi$, whereas $t$ is even. In the most general situation, the CS amplitudes are functions of four independent variables, e.g., the photon energies and virtualities, $A_{i}=A_{i}\left(v, v^{\prime} ; q^{2}, q^{\prime 2}\right)$, or equivalently, $A_{i}\left(\xi, t ; q^{2}, q^{\prime 2}\right)$.

Now, for real photons $\left(q^{2}=q^{\prime 2}=0\right)$, small $v$ infers the smallness of $v^{\prime}$ and $t$. This is the limit in which the static polarizabilities are defined. For both photons virtual and having the same momentum $q^{\prime}=q$, we deal with the forward VVCS process which, by means of unitarity and causality, can be expressed in terms of nucleon structure functions. In this case the low-energy expansion of the non-Born amplitude ${ }^{1}$ is around $v=v^{\prime}=0$ and the polarizabilities arise as moments of structure functions.

In this work we are concerned with VCS - the Compton process where the initial photon is virtual $\left(q^{2}=-Q^{2}\right)$ and the final one is real $\left(q^{\prime 2}=0\right)$. The polarizabilities are obtained by expanding around $v^{\prime}=0$, while $v$ is required to be near the lowest physical value (i.e., the elastic threshold): $v_{0}=Q^{2} / 2 M$. This limit corresponds with $t=-Q^{2}$. Note that our $Q^{2}$ corresponds to $\tilde{Q}^{2}$ in the notation of Ref. [3], and to $Q_{0}^{2}$ in the notation of Ref. [6]. The threemomentum squared of the initial photon is given in this limit by $\overline{\mathrm{q}}^{2}=Q^{2}(1+\tau)$, with $\tau=Q^{2} / 4 M^{2}=v_{0} / 2 M$.

To distinguish between the GPs arising in VVCS and VCS, we shall refer to the former ones as "symmetric" and to the latter ones as "skewed". The three considered situations can thus be classified as follows:

static $(\mathbf{R C S}): q^{2}=0, q^{\prime 2}=0, v \approx 0, v^{\prime} \approx 0, t \approx 0$. symmetric GPs (VVCS): $q^{2}=q^{\prime 2}=-Q^{2}, v=v^{\prime} \approx 0$, $t=0$.

skewed GPs (VCS): $q^{2}=-Q^{2}, q^{\prime 2}=0, v^{\prime} \approx 0, v \approx$ $Q^{2} / 2 M, t \approx-Q^{2}$.

A nice pictorial representation of these situations can be found in Ref. [38]. Because the low-energy expansions in VVCS and VCS are performed around such different kinematical points, the symmetric and skewed GPs are only connected in the static (real-photon) limit.

Let us now consider the low-energy expansion of VCS in more detail. The low-energy theorem for $\operatorname{VCS}[3,39,40]$ states that the expansion of the non-Born amplitude in powers of the final photon's energy $v^{\prime}$ starts at $O\left(v^{\prime 1}\right)$, whereas the two leading terms, $O\left(v^{\prime-1}\right)$ and $O\left(v^{\prime 0}\right)$, are entirely determined by the tree-level $\mathrm{BH}$ and Born amplitudes. The leading-order $\left[O\left(v^{\prime 1}\right)\right]$ non-Born contribution had initially

1 The Born amplitude will be treated exactly, as the convergence of its low-energy expansion is severely limited by the nucleon pole at $\xi= \pm Q^{2} / 2 M$. been parametrized by ten skewed GPs [3]. Soon after, it was discovered that the crossing symmetry [see Eq. (6b)] reduces the number of independent GPs to six [6]. These six GPs are often denoted as ${ }^{2}$

$$
\begin{aligned}
& P^{(L 1, L 1) 0}\left(Q^{2}\right), P^{(M 1, M 1) 0}\left(Q^{2}\right), P^{(L 1, L 1) 1}\left(Q^{2}\right), \\
& P^{(M 1, M 1) 1}\left(Q^{2}\right), P^{(M 1, L 2) 1}\left(Q^{2}\right), P^{(L 1, M 2) 1}\left(Q^{2}\right),
\end{aligned}
$$

where $P^{\left(\rho^{\prime} \ell^{\prime}, \rho \ell\right) S}\left(Q^{2}\right)$ correspond with a multipole amplitude (at $v^{\prime}=0$ ) where $\rho=L, M$ denotes whether the photon is of the longitudinal or the magnetic type and $\ell$ denotes the angular momentum (respectively, $\rho^{\prime} \ell^{\prime}$ or $\rho \ell$ for the final or initial photon); $S=1$ or $S=0$ indicates whether the transition involves the proton's spin flip or not.

To be more specific, we consider the tensor decomposition of the VCS amplitude $\mathcal{M}^{\mu v}$ into the gauge-invariant basis of Ref. [6]:

$\mathcal{M}^{\mu \nu}\left(p^{\prime}, q^{\prime}, p, q\right)=e^{2} \sum_{i=1}^{12} \rho_{i}^{\mu v} A_{i}\left(\xi, t ; q^{2}\right)$,

where $\mu(v)$ are the indices of the outgoing (incoming) photon four-vector fields, with tensors $\rho_{i}$ given in Appendix A. The nucleon crossing symmetry in combination with charge conjugation yields the following property of the invariant amplitudes:

$A_{i}\left(\xi, t ; q^{2}\right)=+A_{i}\left(-\xi, t ; q^{2}\right), \quad i=1,2,5,6,7,9,11,12$

$A_{i}\left(\xi, t ; q^{2}\right)=-A_{i}\left(-\xi, t ; q^{2}\right), \quad i=3,4,8,10$,

with the latter equation leading to the above-mentioned reduction of the number of independent GPs from 10 to 6 . This property is also helpful in checking our loop calculations.

The six GPs of Eq. (4) are defined in terms of the non-Born amplitudes, ${ }^{3}$

$\bar{A}_{i}\left(\xi, t ; q^{2}\right) \equiv A_{i}\left(\xi, t ; q^{2}\right)-A_{i}^{\mathrm{Born}}\left(\xi, t ; q^{2}\right)$,

taken at $\xi=0$ and $t=q^{2} \equiv-Q^{2}$. Introducing a shorthand notation,

$\bar{A}_{i}\left(Q^{2}\right) \equiv \bar{A}_{i}\left(0,-Q^{2} ;-Q^{2}\right)$,

the precise expressions for the GPs are given by

$$
\begin{aligned}
& P^{(L 1, L 1) 0}\left(Q^{2}\right)=\sqrt{\frac{2}{3}} N_{q}\left[\bar{A}_{1}\left(Q^{2}\right)+4 M^{2}(1+\tau) \bar{A}_{2}\left(Q^{2}\right)\right. \\
& \left.+4 M^{2} \tau\left(2 \bar{A}_{6}\left(Q^{2}\right)+\bar{A}_{9}\left(Q^{2}\right)-\bar{A}_{12}\left(Q^{2}\right)\right)\right]
\end{aligned}
$$

$\overline{2 \text { Originally [3] }}$ they were denoted as, respectively: $P^{(01,01) 0}, P^{(11,11) 0}$, $P^{(01,01) 1}, P^{(11,11) 1}, P^{(11,02) 1}, P^{(01,12) 1}$.

3 Expressions for the Born contribution in this basis can for instance be found in Ref. [20]. 


$$
\begin{aligned}
& P^{(M 1, M 1) 0}\left(Q^{2}\right)=-\sqrt{\frac{8}{3}} N_{q} \bar{A}_{1}\left(Q^{2}\right), \\
& P^{(L 1, L 1) 1}\left(Q^{2}\right)=-\frac{2}{3} N_{q} M \tau\left[\bar{A}_{5}\left(Q^{2}\right)+\bar{A}_{7}\left(Q^{2}\right)\right. \\
& \left.+4 \bar{A}_{11}\left(Q^{2}\right)+4 M \bar{A}_{12}\left(Q^{2}\right)\right], \\
& P^{(M 1, M 1) 1}\left(Q^{2}\right)=-\frac{2}{3} N_{q} \frac{M \tau}{1+\tau}\left[\bar{A}_{5}\left(Q^{2}\right)-2 M \tau \bar{A}_{12}\left(Q^{2}\right)\right], \\
& P^{(M 1, L 2) 1}\left(Q^{2}\right)=\frac{2}{3} \sqrt{\frac{2}{3}} N_{q}\left[\frac{\tau}{2(1+\tau)} \bar{A}_{5}\left(Q^{2}\right)\right. \\
& \left.+\frac{1}{2} \bar{A}_{7}\left(Q^{2}\right)+2 \bar{A}_{11}\left(Q^{2}\right)+\frac{M \tau}{1+\tau} \bar{A}_{12}\left(Q^{2}\right)\right], \\
& P^{(L 1, M 2) 1}\left(Q^{2}\right)=-\frac{\sqrt{2}}{6} N_{q} \frac{1}{1+\tau}\left[8 M \bar{A}_{6}\left(Q^{2}\right)+\bar{A}_{7}\left(Q^{2}\right)\right. \\
& \left.+4 M \bar{A}_{9}\left(Q^{2}\right)+4 \bar{A}_{11}\left(Q^{2}\right)+2 M \tau \bar{A}_{12}\left(Q^{2}\right)\right],
\end{aligned}
$$

with the normalization factor $N_{q}$ usually taken to be

$N_{q}=\sqrt{\frac{2 M+v_{0}}{2\left(M+v_{0}\right)}}=\sqrt{\frac{1+\tau}{1+2 \tau}}$.

At the real-photon point, these GPs relate to the static nucleon polarizabilities $[3,7]$ :

$$
\begin{aligned}
P^{(L 1, L 1) 0}(0) & =-\frac{1}{\alpha_{\mathrm{em}}} \sqrt{\frac{2}{3}} \alpha_{E 1}, \\
P^{(M 1, M 1) 0}(0) & =-\frac{1}{\alpha_{\mathrm{em}}} \sqrt{\frac{8}{3}} \beta_{M 1}, \\
P^{(M 1, L 2) 0}(0) & =-\frac{1}{\alpha_{\mathrm{em}}} \frac{2}{3} \sqrt{\frac{2}{3}} \gamma_{M 1 E 2}, \\
P^{(L 1, M 2) 0}(0) & =-\frac{1}{\alpha_{\mathrm{em}}} \frac{\sqrt{2}}{3} \gamma_{E 1 M 2},
\end{aligned}
$$

where $\alpha_{\mathrm{em}}=e^{2} / 4 \pi \simeq 1 / 137$ is the fine structure constant. The remaining two GPs, $P^{(L 1, L 1) 1}$ and $P^{(M 1, M 1) 1}$, vanish (at $Q^{2}=0$ ). Their slopes, on the other hand, can be related to other static polarizabilities and to "symmetric" GPs via the spin-dependent sum rules [41-43].

The "skewed" generalizations of the electric and magnetic dipole polarizabilities are thus defined as follows:

$$
\begin{aligned}
& \alpha_{E 1}\left(Q^{2}\right)=-\alpha_{\mathrm{em}} \sqrt{\frac{3}{2}} P^{(L 1, L 1) 0}\left(Q^{2}\right), \\
& \beta_{M 1}\left(Q^{2}\right)=-\alpha_{\mathrm{em}} \sqrt{\frac{3}{8}} P^{(M 1, M 1) 0}\left(Q^{2}\right) .
\end{aligned}
$$

Similar generalizations can be made for the two spin polarizabilities in Eqs. (11c) and (11d).

Finally, let us recall the relation to experimental observables. As noted above, the six GPs of Eq. (4) suffice to fully parametrize the leading (linear in $v^{\prime}$ ) term in the non-Born
VCS amplitude. The latter, together with the BH and Born VCS amplitudes, can be used to calculate the observables of $e p$ bremsstrahlung. Most notably, the expression for the unpolarized five-fold differential cross-section can be cast in the following form:

$$
\begin{aligned}
\mathrm{d}^{5} \sigma= & \mathrm{d}^{5} \sigma^{\text {BH+Born }}+v^{\prime} \Phi\left\{V_{1}\left[P_{L L}\left(Q^{2}\right)-\frac{1}{\varepsilon} P_{T T}\left(Q^{2}\right)\right]\right. \\
& \left.+V_{2} \sqrt{\varepsilon(1+\varepsilon)} P_{L T}\left(Q^{2}\right)\right\},
\end{aligned}
$$

where $\Phi, V_{1}$, and $V_{2}$ are kinematical factors (see Ref. [3] for the specific expressions thereof), $\varepsilon$ is the electron polarization transfer parameter, $P_{L L}, P_{T T}$, and $P_{L T}$ are the VCS response functions given in terms of GPs as follows [4]:

$$
\begin{aligned}
& P_{L L}\left(Q^{2}\right)=-2 \sqrt{6} M G_{E}\left(Q^{2}\right) P^{(L 1, L 1) 0}\left(Q^{2}\right), \\
& P_{T T}\left(Q^{2}\right)=6 M G_{M}\left(Q^{2}\right)(1+\tau)\left[2 \sqrt{2} M \tau P^{(L 1, M 2) 1}\left(Q^{2}\right)\right. \\
& \left.\quad+P^{(M 1, M 1) 1}\left(Q^{2}\right)\right], \\
& P_{L T}\left(Q^{2}\right)=\sqrt{\frac{3}{2}} M \sqrt{1+\tau}\left[G_{E}\left(Q^{2}\right) P^{(M 1, M 1) 0}\left(Q^{2}\right)\right. \\
& \left.\quad-\sqrt{6} G_{M}\left(Q^{2}\right) P^{(L 1, L 1) 1}\left(Q^{2}\right)\right],
\end{aligned}
$$

where $G_{E}\left(Q^{2}\right)$ and $G_{M}\left(Q^{2}\right)$ are the Sachs electric and magnetic form factors of the nucleon.

The unpolarized differential cross-section thus gives information about two linear combinations of the VCS response functions: $P_{L L}-P_{T T} / \varepsilon$ and $P_{L T}$. These two quantities are dominated by the scalar GPs: $P^{(L 1, L 1) 0}$ and $P^{(M 1, M 1) 0}$. Note, however, that performing unpolarized VCS experiments at fixed $Q^{2}$ and for two different values of $\varepsilon$ allows one to separate $P_{L L}$ and $P_{T T}$ and thus to access one combination of spin GPs, $P_{T T}$. To obtain information on all spin GPs, one needs to consider polarization observables. We will be interested, in particular, in the response function [44]

$$
P_{L T}^{\perp}\left(Q^{2}\right)=\frac{M}{Q} \frac{G_{E}\left(Q^{2}\right)}{G_{M}\left(Q^{2}\right)} P_{T T}\left(Q^{2}\right)-\frac{Q}{4 M} \frac{G_{M}\left(Q^{2}\right)}{G_{E}\left(Q^{2}\right)} P_{L L}\left(Q^{2}\right)
$$

which has been accessed experimentally using the beamrecoil polarization asymmetries [13].

\section{Chiral perturbation theory of generalized polarizabilities}

Our aim is to compute the nucleon VCS amplitudes $A_{i}\left(\xi, t ; q^{2}\right)$ and subsequently the "skewed" GPs using the $\mathrm{SU}(2)$ chiral perturbation theory $(\chi \mathrm{PT})[45,46]$, including the nucleon and $\Delta(1232)$ degrees of freedom. We shall employ $\mathrm{B} \chi \mathrm{PT}$ which is the manifestly covariant extension 
of $\chi \mathrm{PT}$ to the single-baryon sector in its most straightforward implementation (i.e., not the "infrared regularization" of Ref. [22]), where the nucleon is included as in ${ }^{4}$ Ref. [47], and the $\Delta(1232)$ as in Ref. [48]; see also [49] for concise overview. The heavy-baryon results can easily be obtained from B $\chi$ PT by an additional expansion in the inverse powers of baryon masses.

\subsection{Further remarks on power counting}

Let us recall that the chiral effective-field theory is based on the perturbative expansion in powers of pion momentum $p$ and mass $m_{\pi}$ over the scale of spontaneous chiral symmetry breaking $\Lambda_{\chi} \sim 4 \pi f_{\pi}$, with $f_{\pi} \simeq 92 \mathrm{MeV}$ the pion decay constant. Each operator in the effective Lagrangian, or a graph in the loopwise expansion of the S-matrix, can be assigned with an order of $p$. To give a relevant example consider the operator

$\bar{N} N F^{2}$

with $N(x)$ standing for the Dirac field of the nucleon, and $F^{2}$ is the square of the electromagnetic field tensor, $F_{\mu \nu}(x)=$ $\partial_{[\mu} A_{\nu]}(x)$. This is an operator of $O\left(p^{4}\right)$, since two of the $p$ 's come from the photon momenta which are supposed to be small, and the other two powers arise because the two-photon coupling to the nucleon must carry a factor of $\alpha_{\mathrm{em}}$ (the charge $e$ counts as $p$, as we want the derivative of the pion field to count as $p$ even after including the minimal coupling to the photon).

This operator enters the effective Lagrangian with a socalled low-energy constant (LEC), denoted by $C$, and it gives a contribution to the Compton scattering amplitude in the form of 5

$\mathcal{M}_{C}^{\mu \nu}=C\left(q \cdot q^{\prime} g^{\mu \nu}-q^{\mu} q^{\prime v}\right)$,

hence leading to a shift in the magnetic dipole polarizability: $\beta_{M 1} \rightarrow \beta_{M 1}+C / 4 \pi$. Now, two important remarks are in order.

(i) Naturalness. The value of $C$ is not completely arbitrary, but should rather go as $C=\left(e^{2} / \Lambda_{\chi}^{3}\right) c$, with the dimensional constant $c$ being of order of 1 , or more precisely:

$$
p / \Lambda_{\chi} \ll|c| \ll \Lambda_{\chi} / p
$$

\footnotetext{
4 The power counting concerns raised in [47] have been overcome by renormalizing away the "power-counting violating" using the lowenergy constants (a.k.a. Wilson coefficients) available at that order. This has been shown explicitly within the "extended on-mass-shell renormalization scheme" (EOMS) [23], but it is not limited to it.

5 Throughout this paper we use the conventions summarized in the beginning of Ref. [43].
}

This condition ensures that the contribution of this operator is indeed of $O\left(p^{4}\right)$, as the power counting requires.

(ii) Predictive powers. This LEC enters very prominently in Compton scattering and polarizabilities - at the tree level, which means its value is best fixed by the empirical information on these quantities. If this is so, the $O\left(p^{4}\right)$ is not "predictive", as it could only be used to fit the $\chi \mathrm{PT}$ result to experiment or lattice QCD calculations. On the other hand, contributions of orders lower than $p^{4}$ are predictive, as they only contain LECs fixed from elsewhere. It is crucial to first study the predictive contributions, if there are any, and this is what we shall focus on here, for the case of VCS and GPs.

The "predictive" contributions to Compton scattering and polarizabilities had been identified in Ref. [35] and computed for the case of real-Compton scattering therein and for VVCS in [36]. Our present calculation of VCS is quite analogous to those works and hence we refer to them for most of the technical details, such as the expressions for the relevant terms of the effective Lagrangian.

On the conceptual side, it is important to note that the counting of the $\Delta(1232)$ effects is done in the so-called " $\delta$-counting" [50]. In it, the Delta-nucleon mass difference $\Delta=M_{\Delta}-M$ is counted as a light scale $\left(\Delta \ll \Lambda_{\chi}\right)$ which is substantially heavier than the pion mass $\left(m_{\pi} \ll \Delta\right)$. Hence, if $p \sim m_{\pi}$, then $O\left(p^{4} / \Delta\right)$ is in between of $O\left(p^{3}\right)$ and $O\left(p^{4}\right)$.

For the non-Born VCS amplitude and polarizabilities the predictive orders are $O\left(p^{3}\right)$ and $O\left(p^{4} / \Delta\right)$. The $O\left(p^{3}\right)$ contribution comes from the pion-nucleon loops shown in Fig. 3. We refer to it here as the leading-order (LO) contribution. ${ }^{6}$ The $O\left(p^{4} / \Delta\right)$ contribution, arising at the next-to-leading order (NLO), comes from the Delta pole graph and the pionDelta loops shown in Fig. 4.

Going into more detail, we note that the feature of the $\delta$ counting is that the characteristic momentum $p$ distinguishes two regimes: low-energy $\left(p \simeq m_{\pi}\right)$, and resonance $(p \simeq \Delta)$. The above counting is limited to the low-energy regime. Since we are interested in the VCS amplitude at the specific kinematics point where the GPs are defined (i.e., $\xi=0$, $t=-Q^{2}$ ), we do not consider the regime where one-Deltareducible graphs are enhanced (resonance regime). However, going to higher $Q$ one does need to count the Delta propagators similar to the nucleon propagators, which, in turn, calls for inclusion of pion-Delta loops with two and three Delta propagators, which have been omitted here. They are only included implicitly to restore current conservation by

\footnotetext{
${ }^{6}$ In the full Compton amplitude (i.e., including the Born term), it is, in fact, a next-to-leading order contribution, and this is how it is referred to sometimes, e.g. [35].
} 

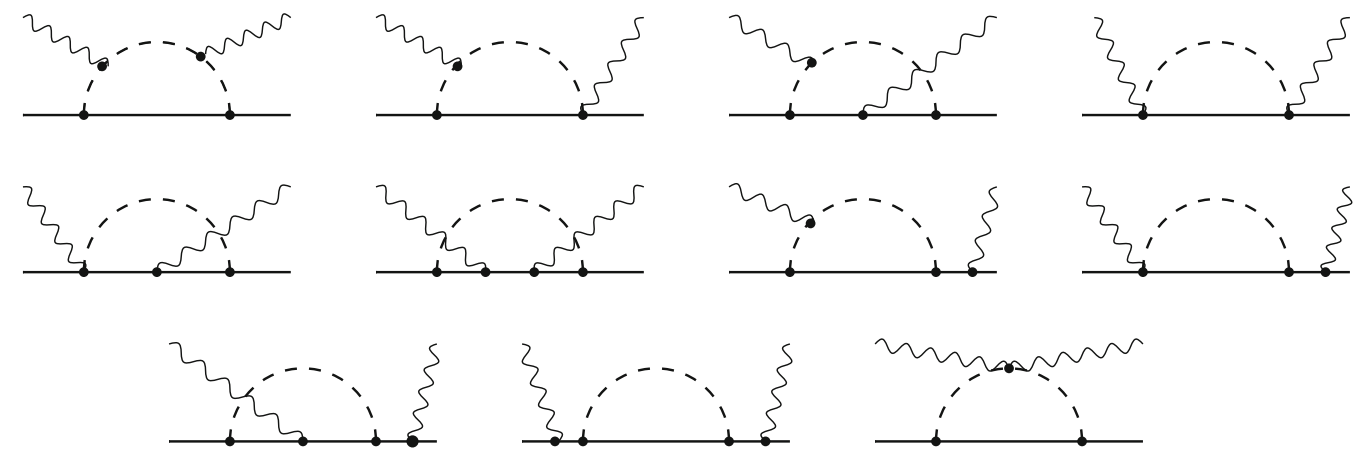

Fig. 3 Pion-nucleon loops of $O\left(p^{3}\right)$. Solid (dashed) lines denote nucleons (pions). Crossed and time-reversed graphs are not shown but are included in the calculation

Fig. 4 Pion-Delta loops and the Delta tree of $O\left(p^{4} / \Delta\right)$ (in the low-energy regime). Crossed and time-reversed graphs are not shown but are included in the calculation. Double lines denote the propagator of the Delta
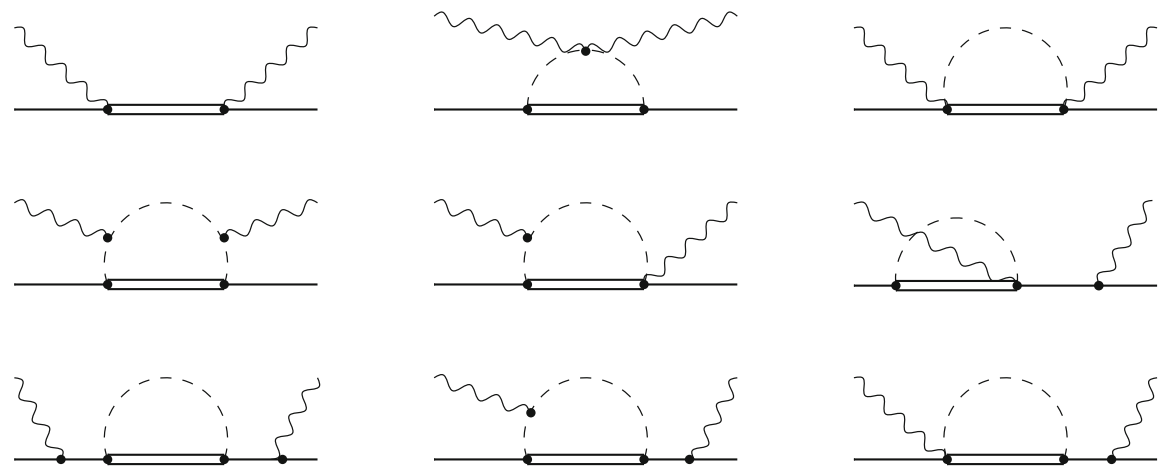

adjusting the isospin coefficients of one-nucleon-reducible graphs in Fig. 4, as explained in Ref. [35]. Apart from that, pion-Delta loops have a rather mild dependence on momenta and the missing loops are unlikely to significantly affect the $Q^{2}$-dependence of the GPs, even for $Q^{2}$ comparable to $\Delta^{2}$.

To conclude this section, a remark is in order about the $\pi^{0}$ anomaly graph, sometimes considered to be a part of the Born contribution. It enters the VCS amplitude at $O\left(p^{2}\right)$ and represents the dominant part of the spin GPs (all except $P^{(L 1, L 1) 1}$ where it does not enter). However, the anomaly contributions cancel in the response functions introduced above. We will also omit them when showing results for the spin GPs.

\subsection{In practice}

The calculation of the $\pi N$ and $\pi \Delta$ loop graphs in Figs. 3 and 4 is analogous to Ref. [35], with the obvious extension to the case of finite virtuality of the initial photon. The renormalization is done in exactly the same way; namely, graphs with the nucleon self-energy and with the one-loop $\gamma N N$ vertices are subtracted according to the usual prescription

$$
\Sigma_{R}\left(\not \not_{s}\right)=\Sigma\left(\not p_{s}\right)-\Sigma(\not p)-\Sigma^{\prime}(\not p)\left(\not p_{s}-M\right),
$$

$\Gamma_{R}^{\mu}\left(p, p^{\prime}\right)=\Gamma^{\mu}\left(p, p^{\prime}\right)-\left[\gamma^{\mu} F_{1}\left(q^{2}\right)-\frac{1}{2 M} \gamma^{\mu \nu} q_{\nu} F_{2}\left(q^{2}\right)\right]$

where $p_{s}$ and $p$ in the first equation are the off-shell and the on-shell momentum of the nucleon, whereas $F_{1}\left(q^{2}\right)$ and $F_{2}\left(q^{2}\right)$ are the on-shell nucleon Dirac and Pauli form factors resulting from the unsubtracted $\gamma N N$ vertex $\Gamma^{\mu}\left(p, p^{\prime}\right)$, with $q=p^{\prime}-p$ being the momentum transfer from the photon to the nucleon.

The Delta pole graph in Fig. 4 is calculated in Refs. [26, 36], and as in those works the magnetic $\gamma N \Delta$ coupling $g_{M}$ acquires the dipole behavior that mimics the form expected from vector-meson dominance:

$g_{M} \rightarrow \frac{g_{M}}{\left[1+(Q / \Lambda)^{2}\right]^{2}}$,

with the dipole mass $\Lambda^{2}=0.71 \mathrm{GeV}^{2}$.

Concerning the implementation of tensor decomposition in Eq. (5), it proved to be useful to write the basis tensors $\rho_{i}, i=1, \ldots, 12$, in terms of the Tarrach tensors $T_{i}, i=1, \ldots, 34$, introduced for the most general VVCS case [51]; see Appendix A. All tensors $\rho_{i}$, apart from $\rho_{2}$, 
$\rho_{3}$, and $\rho_{6}$, have unique structures that allow for unambiguous identification of the corresponding parts of the amplitude, e.g., the combination $T_{29}+T_{30}$ enters only $\rho_{12}, T_{17}$ enters only $\rho_{11}$, and so on. After the tensors $\rho_{i}, i \neq 2,3,6$, have been identified, the remaining tensors can be identified as well, since at this stage they are the only ones that can enter the rest of the amplitude. Since the basis $\rho_{i}$ is explicitly gauge invariant, all the terms that are not proportional to any of $\rho_{i}$ have to vanish when one decomposes a gauge invariant amplitude, e.g., summing up a gauge invariant subset of Feynman graphs such as the $\pi N$ loops in Fig. 3 with their crossed and time-reversed partners, or the Delta pole graph in Fig. 4 with its crossed partner, or the $\pi \Delta$ loops in that figure with their crossed and time-reversed partners. Ensuring that the rest of the amplitude vanishes after the terms proportional to $\rho_{i}$ have been subtracted represents a non-trivial check of a VCS calculation.

We note as well that the tensor decomposition introduces false singularities in the amplitudes due to some of the coefficients in front of $T_{i}$ proportional to $\xi$; these singularities disappear in the end, which serves as yet another check of the calculation. These singularities tend to interfere with the false on-shell singularities in one-nucleon-reducible graphs, i.e., graphs with the nucleon self-energy loop and those with the one-loop $\gamma N N$ vertices. These latter singularities also have to disappear in the end since both the self-energy and the one-loop $\gamma N N$ vertices are subtracted on-shell, as explained above.

It is more convenient, however, to explicitly remove on-shell singularities from the integrals over the Feynman parameters; this can be done by integrating by parts in these integrals and by additional subtractions where they are needed. To illustrate these techniques, we give two examples of typical terms arising in the $\pi N$ self-energy graph, where the on-shell singularities are manifest:

$$
\begin{aligned}
& \text { (A) } X_{1}=\frac{1}{\bar{s}-1} \int_{0}^{1} \mathrm{~d} x\left\{\log \left[x^{2}+\mu^{2}(1-x)-(\bar{s}-1) x(1-x)\right]\right. \\
& \left.-\log \left[x^{2}+\mu^{2}(1-x)\right]\right\}, \\
& \text { (B) } X_{2}=\frac{1}{\bar{s}-1} \int_{0}^{1} \mathrm{~d} x \\
& \times \frac{x^{2}\left[x^{2}(2 x-1)-3 \mu^{2}(x-1)^{2}+2(\bar{s}-1) x(x-1)^{2}\right]}{\left[x^{2}+\mu^{2}(1-x)\right]\left[x^{2}+\mu^{2}(1-x)-(\bar{s}-1) x(1-x)\right]},
\end{aligned}
$$

here $\mu=m_{\pi} / M$ and $\bar{s}=s / M^{2}$. The singularity at $\bar{s} \rightarrow 1$ appearing in $X_{1}$ is canceled when one integrates by parts in the first integral, whereas in order to deal with $X_{2}$ one notices that the integral in it vanishes at $\bar{s} \rightarrow 1$. This means that the integrand of $X_{2}$ can be subtracted at $\bar{s}=1$; the singularity cancels after this subtraction.

Removing the on-shell false singularities explicitly allows one to deal with the remaining $1 / \xi$ false singularities that come from the tensor decomposition by simply expanding the integrals in powers of $\xi$. It appears to be possible to analytically verify that coefficients in front of negative powers of $\xi$ turn to zero after integration over the Feynman parameters, both for the $\pi N$ and $\pi \Delta$ loops.

Given the kinematics of VCS, one is only interested in the $\xi^{0}$ term in the expansion of the thus obtained amplitudes $A_{i}\left(\xi, t ; q^{2}\right)$, while the Mandelstam variable $t$ is also set to $t=q^{2} \equiv-Q^{2}$. The resulting functions $\bar{A}_{i}\left(Q^{2}\right)$ are obtained as integrals over two Feynman parameters; an expansion in $Q^{2}$ around the static point $Q^{2}=0$ allows for the integrals to be taken analytically. Appendix B contains expressions for those linear combinations of $\bar{A}_{i}\left(Q^{2}\right)$ that enter the GPs given by Eq. 4, resulting from the $\pi N$ loop graphs in Fig. 3 and from the Delta pole graph in Fig. 4. The corresponding expressions for the $\pi \Delta$ loops in Fig. 4 are given in the supplementary material to this article.

\subsection{Error estimate}

In making comparison with experimental data, it is important to provide a theoretical uncertainty. In the case of an EFT expansion, the common way to obtain this uncertainty is via the estimate of higher-order contributions. This work employs the following estimate. In the low-momenta regime, where the expansion parameter is $\delta \sim p / \Delta$, our calculation is of the next-to-leading order (NLO). A conservative estimate of the next-to-next-to-leading order (NNLO) contributions would be $\operatorname{error}(f)=\delta^{2} f$, where $f$ is a generic VCS amplitude or response function. It is important to note, however, that the error of the scalar polarizabilities $\alpha_{E 1}\left(Q^{2}\right)$ and $\beta_{M 1}\left(Q^{2}\right)$ in the static limit $Q^{2}=0$ is defined by the error of the corresponding static (real) polarizabilities. This error was argued to be small [37] due to the fact that these polarizabilities are very close at NLO to the results obtained in $\mathrm{B} \chi \mathrm{PT}$ fits to real Compton scattering data [52], and that there are contact terms at NNLO that will in any case compensate changes in $\alpha_{E 1}$ and $\beta_{M 1}$ coming from other higher-order mechanisms. The static errors are estimated as $\operatorname{error}\left(\alpha_{E 1}\right.$, static $) \simeq \operatorname{error}\left(\beta_{M 1}\right.$, static $) \sim$ $0.7 \times 10^{-4} \mathrm{fm}^{3}$ (see Ref. [37]); this translates to the uncertainty of $4.7 \mathrm{GeV}^{2}$ and $2.3 \mathrm{GeV}^{2}$ in $P_{L L}(0)$ and $P_{L T}(0)$, respectively. This static uncertainty has to dominate at very small $Q^{2}$, whereas at larger $Q^{2}$ (still in the lowmomenta regime) the term $\delta^{2} f \sim\left(p^{2} / \Delta^{2}\right) f$ will become more important. In practice, we take the sum of the two values.

The uncertainty estimate in the high-momenta regime works in a similar way. In this regime, $p \gtrsim \Delta$, our calcula- 
tion is at an incomplete leading order (LO), however, we will treat it as an LO calculation as argued above. The expansion parameter in this regime can be one of these,

$\delta=\left\{\frac{m_{\pi}}{\Delta}, \frac{p}{\Lambda_{\chi}}, \frac{\Delta}{\Lambda_{\chi}}\right\}$,

and we take the average value of the three in order to estimate the NLO contribution. In summary, our uncertainty estimate for a VCS amplitude or a response function $f$ is given by

$\operatorname{error}(f)= \begin{cases}\operatorname{error}(f, \text { static })+\frac{p^{2}}{\Delta^{2}} f, & p \lesssim \Delta, \\ \frac{1}{3}\left(\frac{m_{\pi}}{\Delta}+\frac{p}{\Lambda_{\chi}}+\frac{\Delta}{\Lambda_{\chi}}\right) f, & p \gtrsim \Delta,\end{cases}$

where $\operatorname{error}(f$, static) is the static uncertainty discussed above. To obtain smooth bands in the plots, the uncertainties in the different regimes are multiplied by smooth transition functions. One has to note that this error estimate can lead to artifacts such as zero crossings, in the regime $p \gtrsim \Delta$ : the error being proportional to the observable, it can become small or even turn to zero if the latter decreases or vanishes. While we still consider this issue to be tolerable as far as the plots we demonstrate here are concerned, one can see it manifest, for instance, in Figs. 8 and 11 below, where the bands of $P_{L T}$ and $\beta_{M 1}$ shrink at larger values of $Q^{2}$.

\section{Comparison with previous calculations}

In this section, we compare our $\mathrm{B} \chi \mathrm{PT}$ results with previous results obtained in $\mathrm{HB} \chi \mathrm{PT}$, in the linear sigma model, and with fixed- $t$ dispersion relations. Matching our results against those obtained in the former two frameworks provides an important check of our calculation.

\subsection{Linear $\sigma$-model}

The first check is made by comparing our results with the results of Metz and Drechsel [53,54] who calculated the nucleon GPs in the linear sigma model at one-loop level. Their linear sigma model calculation, performed in the limit of infinitely large sigma meson mass, is exactly equivalent to the $O\left(p^{3}\right)$ one-loop pion-nucleon $\mathrm{B} \chi \mathrm{PT}$ result. The easiest way to see it is perhaps to compare the Lagrangian used in Refs. [53,54] with the B $\chi$ PT Lagrangian after the field redefinition done in Ref. [35]. Metz and Drechsel provide only the expressions at $Q^{2}=0$ for the GPs and their derivatives. They also give second derivatives for the two spin-dependent GPs that vanish at $Q^{2}=0$. The expressions for all of the spindependent GPs are in addition expanded in $1 / M$ up to NNLO. Our calculation has been able to reproduces all of their results except one: their expression for $\alpha_{E 1}$ of the proton, which we believe to be due to a typo in the second line of Eq. (17) of
Ref. [53], namely, the first term in the square brackets should read 152 instead of 157.

\subsection{Heavy-baryon expansion}

By expanding our results in powers of $1 / M$ we can check against the $\mathrm{HB} \chi \mathrm{PT}$ calculation of Hemmert et al. [17] that includes the Delta isobar in the $\epsilon$-expansion [55]. We checked that the leading term of the heavy-baryon expansion of our results for the scalar GPs (with the $\pi N$ and $\pi \Delta$ loops corresponding to, respectively, $O\left(p^{3}\right)$ and $O\left(\epsilon^{3}\right)$ result of Hemmert et al.) reproduces the results of Ref. [17].

The spin-dependent GPs have also been calculated in $\mathrm{HB} \chi \mathrm{PT}$ without the Delta isobar up to incomplete $O\left(p^{5}\right)$ in Refs. $[18,19]$. These calculations include $\pi N$ loops with photon couplings to the anomalous magnetic moment (a.m.m.) of the nucleon inside the loop, which appear at $O\left(p^{4}\right)$ and are not included in our calculation. Nevertheless, the heavy-baryon expansion of our results should reproduce their $\mathrm{HB} \chi \mathrm{PT}$ expressions, once the a.m.m. couplings are set to zero. We have reproduced the $\mathrm{HB} \chi \mathrm{PT}$ expressions for $P^{(M 1, M 1) 1}$ and $P^{(L 1, M 2) 1}$, calculated in Ref. [19]. The other two spin-dependent GPs, $P^{(L 1, L 1) 1}$ and $P^{(M 1, L 2) 1}$, are reproducible up to the leading order in $1 / M$; the differences at NLO start in the second non-vanishing terms in the expansion in powers of $Q^{2}$, i.e., the first derivative of $P^{(M 1, L 2) 1}$ with respect to $Q^{2}$, and in the second derivative of $P^{(L 1, L 1) 1}$

Is is important to realize that $P^{(L 1, L 1) 1}$ and $P^{(M 1, L 2) 1}$ at $O\left(p^{5}\right)$ were deduced in Ref. [19] by using the nucleon crossing relations of Eq. (6b), which in $\mathrm{HB} \chi \mathrm{PT}$ do not hold exactly due to the lack of an exact charge conjugation symmetry. The mismatch between the $\mathrm{HB}$ results and ours demonstrates that these crossing relations should not be used in $\mathrm{HB} \chi \mathrm{PT}$ to obtain complete expressions for the terms of higher-order in $1 / M$ expansion.

For completeness, we provide here our results for the $\mathrm{HB}$ expansion of the spin GPs up to NLO in $1 / M$. The LO results are the same as given in Refs. [17-19] and read

$$
P_{\mathrm{HB}, \mathrm{LO}}^{(L 1, L 1) 1}\left(Q^{2}\right)=\frac{g_{A}^{2} Q^{2}}{288 \pi^{2} f_{\pi}^{2} m_{\pi}^{2} M}\left[3-\left(2 w^{2}+1\right) L_{1}(w)\right],
$$

$P_{\mathrm{HB}, \mathrm{LO}}^{(M 1, M 1) 1}\left(Q^{2}\right)=-\frac{g_{A}^{2} Q^{2}}{288 \pi^{2} f_{\pi}^{2} m_{\pi}^{2} M}\left[3-2\left(w^{2}+1\right) L_{1}(w)\right]$,

$P_{\mathrm{HB}, \mathrm{LO}}^{(M 1, L 2) 1}\left(Q^{2}\right)=-\frac{g_{A}^{2}}{72 \sqrt{6} \pi^{2} f_{\pi}^{2} m_{\pi}^{2}} L_{1}(w)$,

$P_{\mathrm{HB}, \mathrm{LO}}^{(L 1, M 2) 1}\left(Q^{2}\right)=-\frac{g_{A}^{2}}{144 \sqrt{2} \pi^{2} f_{\pi}^{2} m_{\pi}^{2}} L_{1}(w)$, 

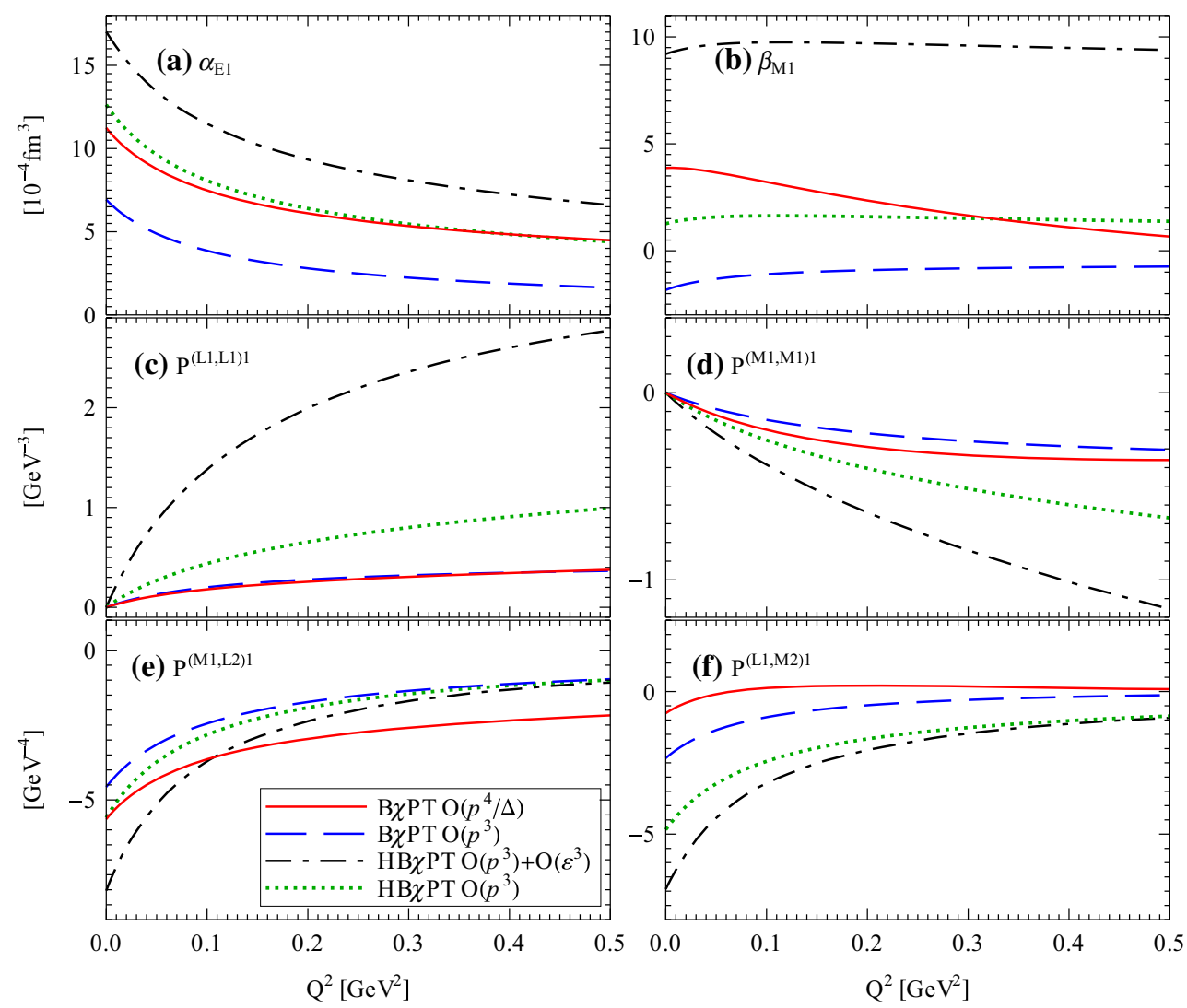

Fig. 5 Comparison of covariant $\mathrm{B} \chi \mathrm{PT}$ and $\mathrm{HB} \chi \mathrm{PT}$ results for generalized polarizabilities: a $\alpha_{E 1}\left(Q^{2}\right)$; b $\beta_{M 1}\left(Q^{2}\right)$; c $P^{(L 1, L 1) 1}\left(Q^{2}\right)$; d $P^{(M 1, M 1) 1}\left(Q^{2}\right)$; e $P^{(M 1, L 2) 1}\left(Q^{2}\right)$; f $P^{(L 1, M 2) 1}\left(Q^{2}\right)$. Red solid curve

with nucleon axial coupling constant $g_{A} \simeq 1.27$, pion decay constant $f_{\pi} \simeq 92.21 \mathrm{MeV}$, where $w=Q / 2 m_{\pi}$, and the function $L_{1}(w)$ is defined as

$L_{1}(w)=\frac{3}{2 w^{2}}\left(1-\frac{\sinh ^{-1} w}{w \sqrt{w^{2}+1}}\right), \quad L_{1}(0)=1$

The correct NLO results (without the nucleon a.m.m. couplings) read

$$
\begin{aligned}
& P_{\mathrm{HB}, \mathrm{NLO}}^{(L 1, L 1) 1}\left(Q^{2}\right)=-\frac{g_{A}^{2} Q^{2}}{2304 \pi f_{\pi}^{2} m_{\pi} M^{2}} \\
& \quad \times\left[\frac{27 w^{2}+30}{w^{2}+1}-\left(9 w^{2}+4\right) L_{2}(w)+\tau_{3}\left(9-\left(3 w^{2}+1\right) L_{2}(w)\right)\right],
\end{aligned}
$$$$
P_{\mathrm{HB}, \mathrm{NLO}}^{(M 1, M 1) 1}\left(Q^{2}\right)=\frac{g_{A}^{2} Q^{2}}{768 \pi f_{\pi}^{2} m_{\pi} M^{2}}
$$$$
\times\left[5-\frac{5 w^{2}+3}{3} L_{2}(w)+\tau_{3}\left(1-\frac{1}{3}\left(w^{2}+1\right) L_{2}(w)\right)\right]
$$

$P_{\mathrm{HB}, \mathrm{NLO}}^{(M 1, L 2) 1}\left(Q^{2}\right)=\frac{g_{A}^{2}}{576 \sqrt{6} \pi f_{\pi}^{2} M m_{\pi}}$
Covariant $O\left(p^{3}\right)+O\left(p^{4} / \Delta\right)$; blue dashed curve covariant $O\left(p^{3}\right)$; black dot-dashed curve $\mathrm{HB} O\left(p^{3}\right)+O\left(\epsilon^{3}\right)$; green dotted curve $\mathrm{HB}$ $O\left(p^{3}\right)$. HB results are from Refs. [17,19]

$$
\begin{gathered}
\times\left[-\frac{3 w^{2}}{w^{2}+1}+\left(w^{2}+2\right) L_{2}(w)+\tau_{3}\left(3+\left(1-w^{2}\right) L_{2}(w)\right)\right], \\
P_{\mathrm{HB}, \mathrm{NLO}}^{(L 1, M 2) 1}\left(Q^{2}\right)=\frac{g_{A}^{2}}{768 \sqrt{2} \pi f_{\pi}^{2} M m_{\pi}} \\
\times\left[\frac{5 w^{2}+7}{w^{2}+1}-\frac{5 w^{2}-3}{3} L_{2}(w)+\tau_{3}\left(1+\frac{1}{3}\left(1-w^{2}\right) L_{2}(w)\right)\right],
\end{gathered}
$$

where

$L_{2}(w)=\frac{3\left(w-\tan ^{-1} w\right)}{w^{3}}, \quad L_{2}(0)=1$,

and $\tau_{3}=+1$ or -1 for the proton or the neutron, respectively.

Our $\mathrm{B} \chi \mathrm{PT}$ results for the GPs are furthermore compared with the analogous $\mathrm{HB} \chi \mathrm{PT}$ results in Fig. 5. Panels (a) and (b) show, respectively, the results for $\alpha_{E 1}\left(Q^{2}\right)$ and $\beta_{M 1}\left(Q^{2}\right)$; one can see that, while the $\operatorname{HB} \chi \mathrm{PT} O\left(p^{3}\right) \pi N$ loops give results very similar to the full $\mathrm{B} \chi \mathrm{PT}$ result (which describes the data quite well, as discussed above), the Delta isobar contribution at $O\left(\epsilon^{3}\right)$ is simply too large to provide a reasonable description of the data. On the other hand, the $\mathrm{B} \chi \mathrm{PT}$ 

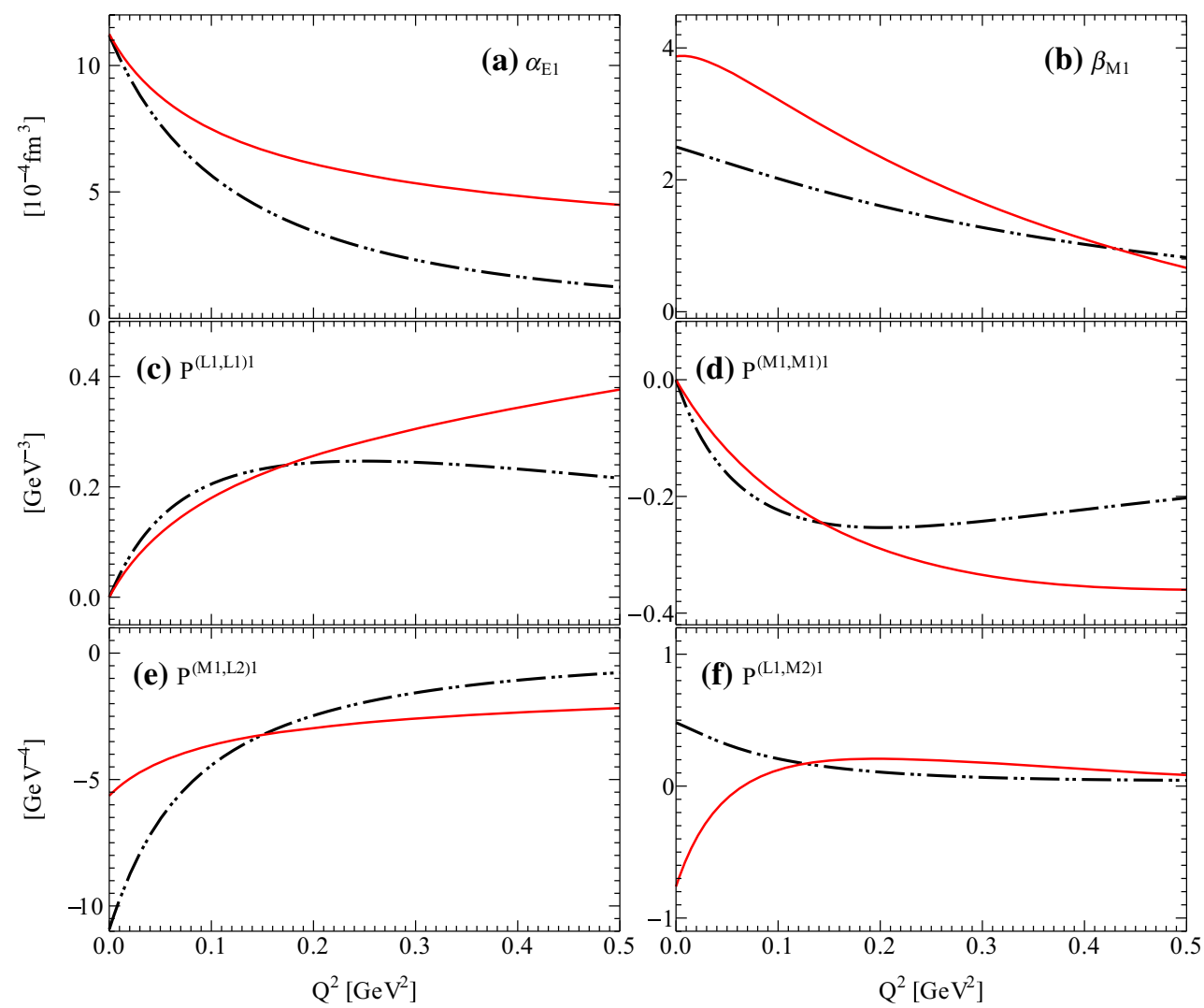

Fig. 6 Comparison of covariant $\mathrm{B} \chi \mathrm{PT}$ and $\mathrm{DR}$ results for generalized polarizabilities: a $\alpha_{E 1}\left(Q^{2}\right) ; \mathbf{b} \beta_{M 1}\left(Q^{2}\right) ; \mathbf{c} P^{(L 1, L 1) 1}\left(Q^{2}\right) ; \mathbf{d} P^{(M 1, M 1) 1}\left(Q^{2}\right)$; e $P^{(M 1, L 2) 1}\left(Q^{2}\right)$; f $P^{(L 1, M 2) 1}\left(Q^{2}\right)$. Red solid curve covariant $\mathrm{B} \chi \mathrm{PT} O\left(p^{3}\right)+O\left(p^{4} / \Delta\right)$; black dash-double-dotted curve $\mathrm{DR}[21]$

$O\left(p^{3}\right) \pi N$ loops underpredict the scalar GPs, which helps to accommodate the Delta isobar contribution at $O\left(p^{4} / \Delta\right)$.

A similar pattern emerges in the case of the spin-dependent GPs, shown in panels (c)-(f); the two GPs that vanish at $Q^{2}=0, P^{(L 1, L 1) 1}$ and $P^{(M 1, M 1) 1}$, are much larger in $\mathrm{HB} \chi \mathrm{PT}$, especially with the Delta isobar. The differences between $\mathrm{B} \chi \mathrm{PT}$ and $\mathrm{HB} \chi \mathrm{PT}$ are perhaps not that large for one of the remaining two GPs, $P^{(M 1, L 2) 1}$, whereas $P^{(L 1, M 2) 1}$ differs more significantly. This can be traced to the values of the spin polarizability $\gamma_{E 1 M 2}$ being different in $\mathrm{B} \chi \mathrm{PT}$ and $\mathrm{HB} \chi \mathrm{PT}$; one has to note, however, that this pattern will change once higher orders in the expansion are included (see also the discussion below).

\subsection{Fixed- $t$ dispersion relations}

We finally compare our results with the calculations based on fixed- $t$ dispersion relations (DR) for the VCS amplitudes [21]. In Fig. 6 we compare the numerical results for the proton GPs. The fixed- $t$ DR calculations rely on the empirical input of pion electro-production multipoles. We compare here with the updated results of Ref. [21] based on the MAID-2007 [56] pion electro-production multipole analysis.
Panel (a) of Fig. 6 shows the electric polarizability, for which one can see a very good agreement at $Q^{2}=0$, which quickly worsens with increasing $Q^{2}$. For the magnetic polarizability, one sees quite an opposite picture; see panel (b). The current PDG value for the static magnetic polarizability, $\beta_{M 1}=2.5(4) \times 10^{-4} \mathrm{fm}^{3}$, is adopted in the fixed- $t$ DR result. Our $\mathrm{B} \chi \mathrm{PT}$ prediction is substantially larger [35,37]: $\beta_{M 1}=3.9(7)$ in the usual units. Fits of Compton scattering data based on $\chi \mathrm{PT}$ also tend to yield a larger value $[52,57]$ : $\beta_{M 1} \simeq 3.2(5)$.

As for higher $Q^{2}$, the $t$ DR calculation of Ref. [21] imposes a dipole fall-off of the subtraction function in the scalar polarizabilities:

$\alpha_{E 1}^{\mathrm{DR}}\left(Q^{2}\right)=\alpha_{E 1}^{\pi N}\left(Q^{2}\right)+\frac{\alpha_{E 1}-\alpha_{E 1}^{\pi N}}{\left[1+Q^{2} / \Lambda_{\alpha}^{2}\right]^{2}}$,
$\beta_{M 1}^{\mathrm{DR}}\left(Q^{2}\right)=\beta_{M 1}^{\pi N}\left(Q^{2}\right)+\frac{\beta_{M 1}-\beta_{M 1}^{\pi N}}{\left[1+Q^{2} / \Lambda_{\beta}^{2}\right]^{2}}$,

where $\alpha_{E 1}^{\mathrm{DR}}\left(Q^{2}\right)$ is the full DR result, $\alpha_{E 1}^{\pi N}\left(Q^{2}\right)$ is the $\pi N$ contribution, with $\alpha_{E 1}$ and $\alpha_{E 1}^{\pi N}$ being the corresponding values at $Q^{2}=0$, with the analogous definitions for $\beta_{M 1}\left(Q^{2}\right)$. In using the $t$ DR results we fix the static values of $\left\{\alpha_{E 1}, \beta_{M 1}\right\}$ 
Table 1 Values of proton mixed spin polarisabilities $\gamma_{E 1 M 2}$ and $\gamma_{M 1 E 2}$, in units of $10^{-4} \mathrm{fm}^{4}$ resulting in the different frameworks: $O\left(p^{4} / \Delta\right)$ $\mathrm{B} \chi \mathrm{PT}$ [37], fixed- $t$ DR [21,58] based on the MAID-2007 [56] multipoles, and $O\left(p^{4}\right) \mathrm{HB} \chi \mathrm{PT}[59,60]$, compared with the latest empirical extraction from experimental data [61]

\begin{tabular}{lll}
\hline Source & $\gamma_{M 1 E 2}$ & $\gamma_{E 1 M 2}$ \\
\hline B $\chi$ PT [37] & $1.1 \pm 0.3$ & $0.2 \pm 0.2$ \\
Fixed- $t$ DR [21,58] & 2.2 & -0.1 \\
HB $\chi$ PT [59,60] & $1.9 \pm 0.5$ & $-0.4 \pm 0.6$ \\
MAMI 2015 [61] & $1.99 \pm 0.29$ & $-0.7 \pm 1.2$ \\
\hline
\end{tabular}

to the current PDG values $\{11.2,2.5\} \times 10^{-4} \mathrm{fm}^{3}$, whereas the cut-offs $\left\{\Lambda_{\alpha}, \Lambda_{\beta}\right\}=\{0.631 \pm 0.011,0.745 \pm 0.021\} \mathrm{GeV}$ are taken from the recent fit of VCS data [14].

For the spin polarizabilities, the GPs $P^{(L 1, L 1) 1}$ and $P^{(M 1, M 1) 1}$ (panels (c) and (d) of Fig. 6, respectively), which vanish for real photons, show good agreement between $\mathrm{B} \chi \mathrm{PT}$ and DR, especially at low $Q^{2}$. The agreement for $P^{(M 1, L 2) 1}$ and $P^{(L 1, M 2) 1}$, shown in panels (e) and (f) of that figure, is not so good. Especially for $P^{(L 1, M 2) 1}$ one notices a different slope at $Q^{2}=0$ between the $\mathrm{B} \chi \mathrm{PT}$ and DR results. On the other hand, $P^{(M 1, L 2) 1}$ and $P^{(L 1, M 2) 1}$ correspond, in the limit $Q^{2}=0$, to the two mixed spin polarizabilities $\gamma_{M 1 E 2}$ and $\gamma_{E 1 M 2}$ (see Eqs. (11c)-(11d)). The former is about two times larger in DR than in $\mathrm{B} \chi \mathrm{PT}$ [37], which would explain the differences in $P^{(M 1, L 2) 1}$ at low $Q^{2}$. The second is small and not well constrained, which means that the difference between $\mathrm{DR}$ and $\mathrm{B} \chi \mathrm{PT}$ is probably not a very serious issue at this stage. To further illustrate this point, we show in Table 1 the values of the two mixed polarizabilities, $\gamma_{M 1 E 2}$ and $\gamma_{E 1 M 2}$, resulting in $\mathrm{B} \chi \mathrm{PT}$ framework at $O\left(p^{4} / \Delta\right)$, in fixed- $t \mathrm{DR}$, in $\mathrm{HB} \chi \mathrm{PT}$ at $O\left(p^{4}\right)$, and the results of extraction of the spin polarizabilities from experimental data of one of the beamtarget asymmetries, $\Sigma_{2 x}$.

\section{Results for VCS observables}

The experiments aiming to measure the GPs are based on the low-energy expansion of the ep $\gamma$ process, Eq. (13), which results in the extraction of the VCS response functions. Then, with some further assumptions on the size of spin GPs, taken usually from the fixed- $t$ DR framework of Ref. [21], one obtains the two scalar GPs, $\alpha_{E 1}$ and $\beta_{M 1}$. We first consider our results at the level of the response functions, since it provides a more direct comparison to experiment.

In Figs. 7, 8, 9 and 10, we show our B $\chi$ PT results (red solid line, with cyan band indicating the uncertainty estimate), compared with the fixed- $t$ DR calculation (blue bands), and experimental data where available. In this calculation we used the Bradford et al. [64] parametrization of nucleon form

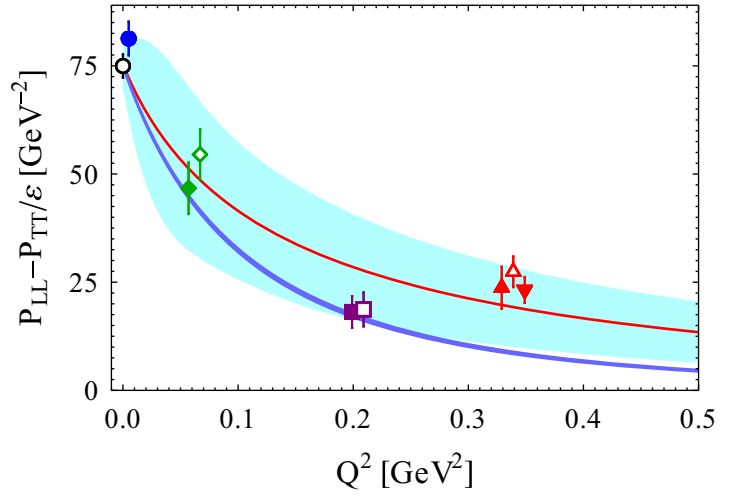

Fig. 7 VCS response function $P_{L L}\left(Q^{2}\right)-P_{T T}\left(Q^{2}\right) / \varepsilon$. The total $O\left(p^{3}\right)+O\left(p^{4} / \Delta\right)$ result is given by the red solid curve with the cyan band showing the estimated theoretical uncertainty as explained in the text. DR results [21] are shown by the blue band. The curves correspond to $\varepsilon=0.65$. The data shown are: black open circle, PDG 2014 [62]; blue circle, Olmos de León et al. [63]; green diamond, MITBates (DR) [8,9]; green open diamond, MIT-Bates (LEX) [8,9]; purple solid square, MAMI (DR) [14]; purple open square, MAMI (LEX) [14]; red solid triangle, MAMI1 (LEX) [10]; red solid inverted triangle, MAMI1 (DR) [12]; red open triangle, MAMI2 (LEX) [11]. Some of the data points are shifted to the right in order to enhance their visibility; namely, Olmos de León, MIT-Bates (LEX), MAMI LEX, MAMI1 DR and MAMI2 LEX sets have the same values of $Q^{2}$ as PDG, MIT-Bates (DR), MAMI DR, and MAMI1 LEX, respectively

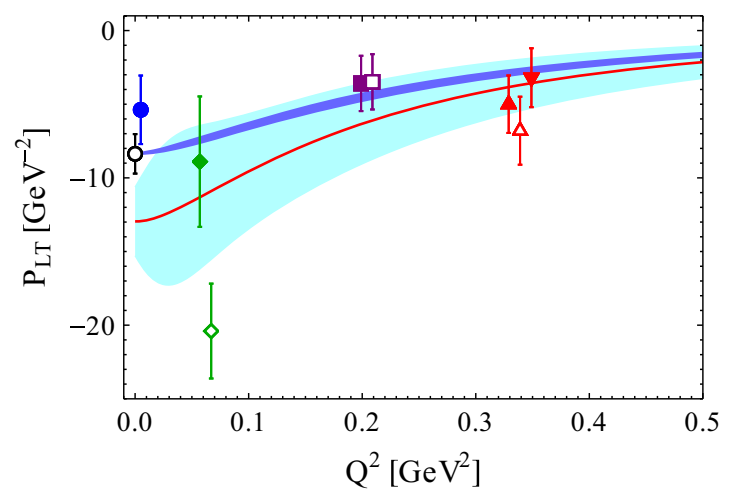

Fig. 8 VCS response function $P_{L T}\left(Q^{2}\right)$. Notation is as in Fig. 7

factors, as input in Eq. (14). The bands of the DR results are obtained by varying the dipole cut-offs $\Lambda_{\alpha}$ and $\Lambda_{\beta}$ within the uncertainties given in Sect. 4.3.

The first two response functions, $P_{L L}-P_{T T} / \varepsilon$ and $P_{L T}$ (Figs. 7,8), are used to extract $\alpha_{E 1}\left(Q^{2}\right)$ and $\beta_{M 1}\left(Q^{2}\right)$, respectively. Our results here are in good agreement with the data as well as with the DR results. The only place of disagreement is $P_{L T}(0)=-2 M \beta_{M 1} / \alpha_{\mathrm{em}}$, due to the larger value of the static magnetic polarizability resulting in $\mathrm{B} \chi \mathrm{PT}$, as mentioned already in the previous section.

Apart from these two response functions extracted from unpolarized measurements, there has been a single low- $Q^{2}$ double-polarization experiment at MAMI [13] extracting the response function $P_{L T}^{\perp}$ defined in Eq. (15). This data point, 


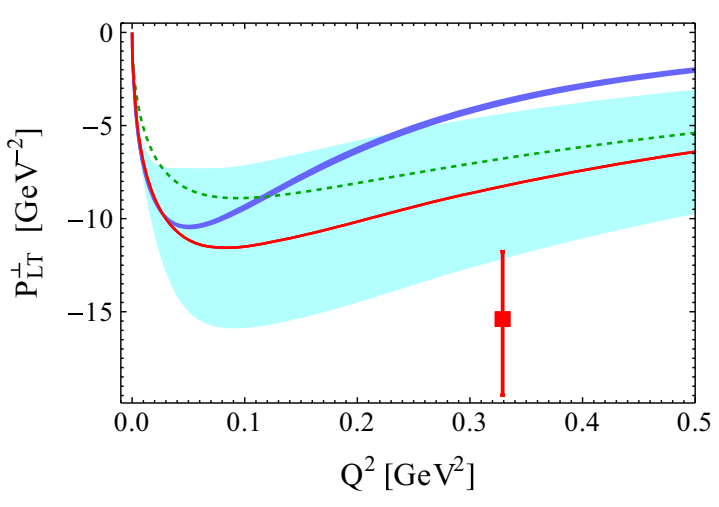

Fig. 9 VCS response function $P_{L T}^{\perp}\left(Q^{2}\right)$. Notation is as in Fig. 7, except from the data: red square, MAMI [13], and the green dotted curve that shows the $\mathrm{B} \chi \mathrm{PT}$ result with only the contribution of $P_{L L}$ included; see Eq. (15)

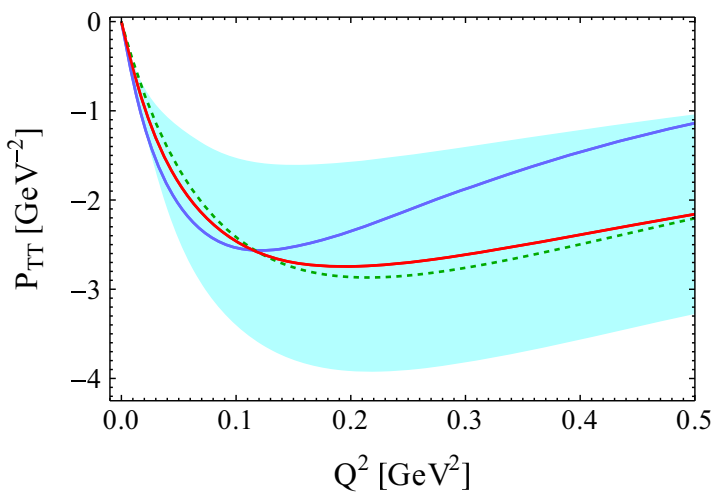

Fig. 10 VCS response function $P_{T T}\left(Q^{2}\right)$. Notation is as in Fig. 7, except from the green dotted curve that shows the $\mathrm{B} \chi \mathrm{PT}$ result with only the contribution of $P^{(M 1, M 1) 1}$ in Eq. (14b)

together with theoretical curves, is shown in Fig. 9. This is perhaps the only place where one can see that the $\mathrm{B} \chi \mathrm{PT}$ calculation is in a better agreement with the data than the DR calculation. On the other hand, the slope at $Q^{2}=0$ is in a perfect agreement between the two calculations.

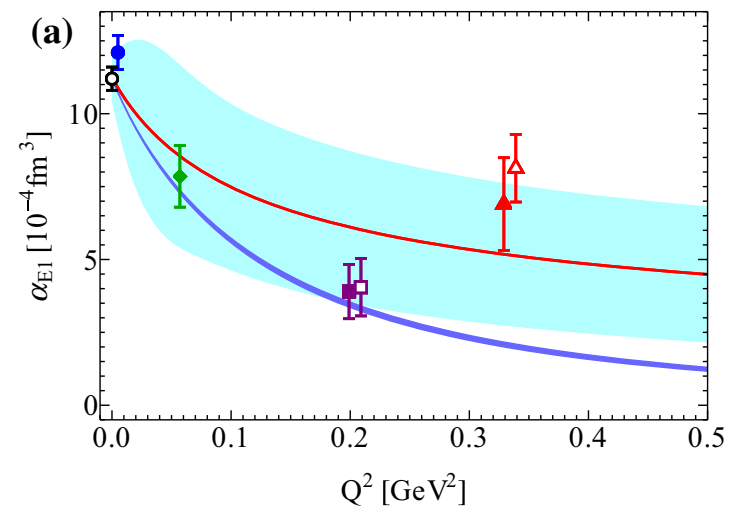

This polarized observable can potentially provide an access to the spin GPs. For instance, combining it with $P_{L L}-P_{T T} / \varepsilon$ one can extract the $P_{T T}$ response function, Fig. 10. The latter is given entirely by the spin GPs. We note that in the $P_{T T}$ response function the large, and well-known, $\pi^{0} t$-channel pole contribution to several of the spin GPs drops out. We see from Fig. 10 that the $\mathrm{B} \chi \mathrm{PT}$ and DR results for $P_{T T}$ are again in reasonable agreement.

In Figs. 9 and 10, we also show (by the green dashed curves) the dominant terms in $P_{L T}^{\perp}$ and in $P_{T T}$. They are given by, respectively, $P_{L L}$ and $P^{(M 1, M 1) 1}$ terms in Eqs. (15) and (14b). One thus sees, in particular, that $P^{(L 1, M 2) 1}$, for which the $\mathrm{B} \chi \mathrm{PT}$ and DR results differ sizably at low $Q^{2}$, gives a very small contribution to $P_{T T}$.

\section{Concluding remarks}

The $\mathrm{B} \chi \mathrm{PT}$ calculation of the nucleon GPs and VCS response functions, presented here, is done to NLO in the $\delta$-counting scheme. It shows a good description of the low- $Q$ data and mostly agrees with the results of the fixed- $t$ DR calculation of Pasquini et al. [20]. The results for the scalar GPs are summarized in Fig. 11, where panel (a) shows the electric polarizability and panel (b) the magnetic one. The theoretical uncertainty of our calculation is sufficiently large to agree with all the data, including the new [14] and old $[10,11]$ MAMI data that tend to disagree among themselves. We can see that the DR curve agrees with the new MAMI data very well, while missing the older data, especially for $\alpha_{E 1}$. For $\beta_{M 1}$, there is an interesting tension at low $Q$ between the DR and $\chi \mathrm{PT}$ results. The available VCS data do not have the necessary precision to resolve the discrepancy.

By making the heavy-baryon expansion we reproduce some of the previous $\mathrm{HB} \chi \mathrm{PT}$ results and, similarly to what was observed in the calculation of the real CS, we find that

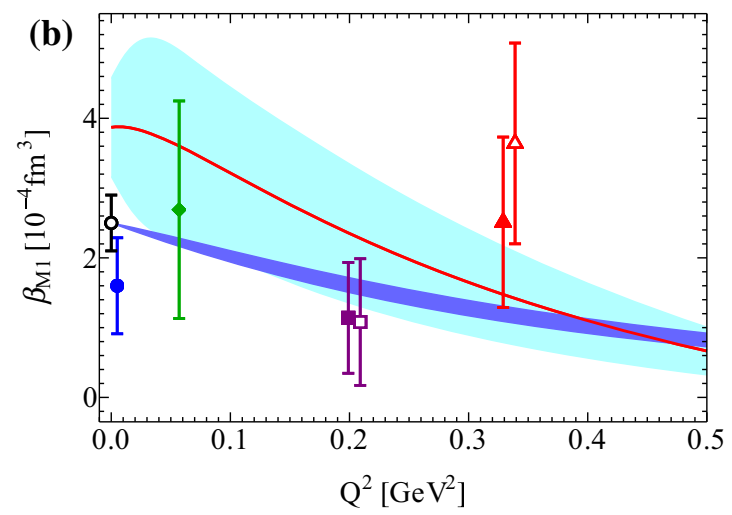

Fig. 11 Generalized scalar polarizabilities: a $\alpha_{E 1}\left(Q^{2}\right), \mathbf{b} \beta_{M 1}\left(Q^{2}\right)$. Description of curves and points is the same as in Fig. 7 
treating the leading chiral loops exactly allows for a more natural accommodation of the Delta-resonance contribution, which is especially large in the magnetic polarizability $\beta_{M 1}$.

We would like to note that a newly approved experiment at Jefferson Lab [65] which plans to measure the unpolarized GPs at $Q^{2}=0.3 \mathrm{GeV}^{2}$ and $Q^{2}=0.75 \mathrm{GeV}^{2}$ will be able to shed further light on the situation. Furthermore, comparing such data at the same $Q^{2}$ value taken at different values of $\varepsilon$ (corresponding with different beam energies) has the potential to separate off the response function $P_{T T}$ in Eq. (13). This would allow one to experimentally access the dominant spin GP $P^{(M 1, M 1) 1}$ for the first time and provide a strong test of the $\mathrm{B} \chi \mathrm{PT}$ predictions presented in this work.

Additionally, new data on the unpolarized response functions and GPs are expected to arrive soon from MAMI. These data will complement the $Q^{2}=0.2 \mathrm{GeV}^{2}$ points [14]. In particular, expected are data at $Q^{2}=0.1 \mathrm{GeV}^{2}$ and $Q^{2}=0.45 \mathrm{GeV}^{2}$, which is in the domain of applicability of $\mathrm{B} \chi \mathrm{PT}$. These data will also further test the theoretical predictions.

One has to admit that the current theoretical uncertainty estimate gives a rather sizeable error band, which should be improved upon. An $O\left(p^{4}\right)$ calculation of GPs in B $\chi \mathrm{PT}$ that would include the remaining $\pi \Delta$ loops that contribute at $O\left(p^{3}\right)$ in the high-momenta regime and both the $\pi N$ and the $\pi \Delta O\left(p^{4}\right)$ contributions in this regime would allow one to significantly decrease the theoretical uncertainty.

Acknowledgements We thank Hélène Fonvieille, Misha Gorchtein, Chungwen Kao, and Barbara Pasquini for stimulating discussions and helpful communications. This work was supported by the Deutsche Forschungsgemeinschaft (DFG) through the Collaborative Research Center "The Low-Energy Frontier of the Standard Model" (SFB 1044) and the Cluster of Excellence PRISMA. V. L. acknowledges partial support of this work by the Moscow Engineering Physics Institute Academic Excellence Project (Contract No. 02.a03.21.0005). We acknowledge the use of FORM [66] in the calculations and of JaxoDraw [67] in preparation of the figures.

Open Access This article is distributed under the terms of the Creative Commons Attribution 4.0 International License (http://creativecomm ons.org/licenses/by/4.0/), which permits unrestricted use, distribution, and reproduction in any medium, provided you give appropriate credit to the original author(s) and the source, provide a link to the Creative Commons license, and indicate if changes were made. Funded by SCOAP ${ }^{3}$.

\section{Appendix A: Tensor decomposition of the VCS amplitude}

In this section we give the details of the tensor decomposition of the VCS amplitude. The basis used by us is $\rho_{i}$, $i=1, \ldots, 12$, introduced in Ref. [6]. Its decomposition in terms of Tarrach's $T_{1}, \ldots, T_{34}$ (which are given below) reads

$$
\begin{aligned}
\rho_{1}= & -q \cdot q^{\prime} T_{1}+T_{3}, \\
\rho_{2}= & -4 M^{2} \xi^{2} T_{1}-4 q \cdot q^{\prime} T_{6}+4 M \xi T_{7}, \\
\rho_{3}= & -2 M \xi Q^{2} T_{1}-M \xi\left(T_{4}+T_{5}\right) \\
& +Q^{2}\left(T_{7}-T_{8}\right)+q \cdot q^{\prime}\left(T_{9}-T_{10}\right), \\
\rho_{4}= & 8 T_{16}-4 M \xi T_{21}+M \xi T_{34}, \\
\rho_{5}= & \frac{1}{2}\left(T_{19}-T_{20}\right)-\frac{Q^{2}}{2} T_{22}-\frac{M \xi}{2}\left(T_{23}+T_{24}\right)-\frac{Q^{2}}{8} T_{34}, \\
\rho_{6}= & -8 q \cdot q^{\prime} T_{6}+4 M \xi T_{7}+4 M q \cdot q^{\prime} T_{21}-4 M^{2} \xi T_{25} \\
& -2 M \xi\left(T_{32}+q \cdot q^{\prime} T_{33}\right)+M q \cdot q^{\prime} T_{34}, \\
\rho_{7}= & T_{18}-q \cdot q^{\prime} T_{22}+M \xi T_{26}, \\
\rho_{8}= & \frac{M \xi}{2}\left(T_{4}+T_{5}\right)+\frac{Q^{2}}{2} T_{8}-\frac{q \cdot q^{\prime}}{2}\left(T_{9}-T_{10}\right) \\
& -\frac{M}{2}\left(T_{14}+T_{15}\right)+\frac{M q \cdot q^{\prime}}{2}\left(T_{23}+T_{24}\right)+\frac{M Q^{2}}{2} T_{26} \\
& +\frac{Q^{2}}{4}\left(T_{32}+q \cdot q^{\prime} T_{33}\right), \\
\rho_{9}= & 2 M \xi T_{8}-2 M q \cdot q^{\prime} T_{22}+2 M^{2} \xi T_{26}-q \cdot q^{\prime} T_{27}+M \xi T_{31}, \\
\rho_{10}= & 2 T_{7}+4 M T_{11}-2 M T_{25}-4 M \xi T_{1}+\left(T_{32}+q \cdot q^{\prime} T_{33}\right), \\
\rho_{11}= & 4 T_{17}-4 M \xi T_{25}+q \cdot q^{\prime} T_{34}, \\
\rho_{12}= & 2 Q^{2} T_{6}+M \xi\left(T_{9}-T_{10}\right)-M Q^{2}\left(T_{21}+T_{22}\right) \\
& -M^{2} \xi\left(T_{23}+T_{24}\right)-\frac{Q^{2}}{2}\left(T_{27}-M \xi T_{33}\right) \\
& -\frac{M \xi}{2}\left(T_{29}+T_{30}\right)-\frac{M Q^{2}}{4} T_{34} .
\end{aligned}
$$

These tensors correspond to the following combinations of Tarrach's $\tau_{i}$ (with $q^{\prime 2}$ set to zero in the latter):

$$
\begin{array}{llll}
\rho_{1}=-\tau_{1}, & \rho_{2}=-4 \tau_{3}, & \rho_{3}=\tau_{4}-\tau_{5}, & \rho_{4}=\tau_{7}, \\
\rho_{5}=\frac{1}{2}\left(\tau_{8}-\tau_{9}\right), & \rho_{6}=\tau_{10}, & \rho_{7}=\tau_{11}, & \rho_{8}=\frac{1}{2}\left(\tau_{12}+\tau_{13}\right), \\
\rho_{9}=\tau_{14}, & \rho_{10}=\tau_{17}, & \rho_{11}=\tau_{18}, & \rho_{12}=\frac{1}{2}\left(\tau_{20}+\tau_{21}\right) .
\end{array}
$$

All tensors apart from $\rho_{2}, \rho_{3}$, and $\rho_{6}$ have unique structures that allow for unambiguous identification, e.g., the combination $T_{29}+T_{30}$ enters only $\rho_{12}, T_{17}$ enters only $\rho_{11}$, and so on. After the tensors $\rho_{i}, i \neq 2,3,6$, have been identified, the remaining tensors can be identified as well. Since this basis is explicitly gauge invariant, all the terms that are not proportional to any of $\rho_{i}$ have to vanish when one decomposes a gauge invariant amplitude, e.g., summing up a gauge invariant subset of Feynman graphs.

The tensors $T_{1}, \ldots, T_{34}$ introduced by Tarrach [51] in order to decompose the CS amplitude in the most general case, i.e., when both $q^{2}$ and $q^{\prime 2}$ are non-zero, are given below; these structures are understood to be contracted with $\epsilon^{v}$ and $\epsilon^{* \mu}$, the incoming and the outgoing photons' polarization vectors. 


$$
\begin{aligned}
& T_{1}=g_{\mu \nu}, T_{17}=\left(P_{\nu} q_{\mu}+P_{\mu} q_{\nu}^{\prime}\right) \mathbb{K}, \\
& T_{2}=q_{\nu} q_{\mu}^{\prime}, T_{18}=\left(P_{\nu} q_{\mu}-P_{\mu} q_{\nu}^{\prime}\right) \not K, \\
& T_{3}=q_{\nu}^{\prime} q_{\mu}, T_{19}=\left(P_{\nu} q_{\mu}^{\prime}+P_{\mu} q_{\nu}\right) \not K, \\
& T_{4}=q_{\nu} q_{\mu}+q_{\nu}^{\prime} q_{\mu}^{\prime}, T_{20}=\left(P_{\nu} q_{\mu}^{\prime}-P_{\mu} q_{\nu}\right) \not K, \\
& T_{5}=q_{\nu} q_{\mu}-q_{\nu}^{\prime} q_{\mu}^{\prime}, T_{21}=P_{\nu} \gamma_{\mu}+P_{\mu} \gamma_{\nu}, \\
& T_{6}=P_{\nu} P_{\mu}, T_{22}=P_{\nu} \gamma_{\mu}-P_{\mu} \gamma_{\nu}, \\
& T_{7}=P_{\nu} q_{\mu}+P_{\mu} q_{\nu}^{\prime}, T_{23}=q_{\nu} \gamma_{\mu}+q_{\mu}^{\prime} \gamma_{\nu}, \\
& T_{8}=P_{\nu} q_{\mu}-P_{\mu} q_{\nu}^{\prime}, T_{24}=q_{\nu} \gamma_{\mu}-q_{\mu}^{\prime} \gamma_{\nu}, \\
& T_{9}=P_{\nu} q_{\mu}^{\prime}+P_{\mu} q_{\nu}, T_{25}=q_{\nu}^{\prime} \gamma_{\mu}+q_{\mu} \gamma_{\nu}, \\
& T_{10}=P_{\nu} q_{\mu}^{\prime}-P_{\mu} q_{\nu}, T_{26}=q_{\nu}^{\prime} \gamma_{\mu}-q_{\mu} \gamma_{\nu}, \\
& T_{11}=g_{\mu \nu} K, T_{27}=2\left(P_{\nu} \gamma_{\mu \lambda} K^{\lambda}+P_{\mu} \gamma_{\nu \lambda} K^{\lambda}\right), \\
& T_{12}=q_{\nu} q_{\mu}^{\prime} K, T_{28}=2\left(P_{\nu} \gamma_{\mu \lambda} K^{\lambda}-P_{\mu} \gamma_{\nu \lambda} K^{\lambda}\right), \\
& T_{13}=q_{\nu}^{\prime} q_{\mu} K, T_{29}=2\left(q_{\nu} \gamma_{\mu \lambda} K^{\lambda}+q_{\mu}^{\prime} \gamma_{\nu \lambda} K^{\lambda}\right), \\
& T_{14}=\left(q_{\nu} q_{\mu}+q_{\nu}^{\prime} q_{\mu}^{\prime}\right) K, T_{30}=2\left(q_{\nu} \gamma_{\mu \lambda} K^{\lambda}-q_{\mu}^{\prime} \gamma_{\nu \lambda} K^{\lambda}\right), \\
& T_{15}=\left(q_{\nu} q_{\mu}-q_{\nu}^{\prime} q_{\mu}^{\prime}\right) K, T_{31}=2\left(q_{\nu}^{\prime} \gamma_{\mu \lambda} K^{\lambda}+q_{\mu} \gamma_{\nu \lambda} K^{\lambda}\right), \\
& T_{16}=P_{\nu} P_{\mu} K, T_{32}=2\left(q_{\nu}^{\prime} \gamma_{\mu \lambda} K^{\lambda}-q_{\mu} \gamma_{\nu \lambda} K^{\lambda}\right), \\
& T_{33}=2 \gamma_{\nu \mu}, \\
& T_{34}=2\left\{\gamma_{\nu \mu}, K K\right\}=4 \gamma_{\nu \mu \lambda} K^{\lambda} .
\end{aligned}
$$

Here, $P=\frac{1}{2}\left(p+p^{\prime}\right), K=\frac{1}{2}\left(q+q^{\prime}\right), \gamma^{\mu \nu}=\frac{1}{2}\left[\gamma^{\mu}, \gamma^{\nu}\right]$ and $\gamma^{\mu \nu \lambda}=\frac{1}{2}\left\{\gamma^{\mu \nu}, \gamma^{\lambda}\right\}$. The following relations hold between these tensors that allow one to exclude two of them (the usual choice being $T_{13}$ and $T_{28}$ ):

$$
\begin{aligned}
& 2\left(T_{17}-T_{19}\right)-\left(q^{2}-q^{\prime 2}\right) T_{22}+2 P \cdot K\left(T_{23}-T_{25}\right) \\
& \quad-2 M T_{28}-2 M P \cdot K T_{32} \\
& +\left(M^{2}+\frac{q \cdot q^{\prime}}{4}-\frac{q^{2}+q^{\prime 2}}{4}\right) T_{34}=0, \quad \text { (A4) } \\
& P \cdot K\left(T_{2}-T_{3}\right)+\frac{1}{4}\left(q^{2}+q^{\prime 2}+2 q \cdot q^{\prime}\right)\left(T_{7}-T_{9}\right) \\
& -\frac{q^{2}-q^{\prime 2}}{4}\left(T_{8}+T_{10}\right)-M\left(T_{12}-T_{13}\right) \\
& +\frac{M}{4}\left(q^{2}+q^{\prime 2}+2 q \cdot q^{\prime}\right)\left(T_{23}-T_{25}\right)-M \frac{q^{2}-q^{\prime 2}}{4}\left(T_{24}+T_{26}\right) \\
& -P \cdot K T_{28}+\frac{q^{2}-q^{\prime 2}}{8}\left(T_{29}-T_{31}\right) \\
& -\frac{1}{8}\left(q^{2}+q^{\prime 2}-2 q \cdot q^{\prime}\right)\left(T_{30}+T_{32}\right) \\
& -\left[(P \cdot K)^{2}+\frac{1}{4}\left(q^{2} q^{\prime 2}-\left(q \cdot q^{\prime}\right)^{2}\right)\right] T_{33}+\frac{M}{2} P \cdot K T_{34}=0 .
\end{aligned}
$$

Taking into account the fact that, for the (real) final photon $\epsilon^{\prime} \cdot q^{\prime}=0$, one can obtain the following useful identities:

$$
T_{2}=0, \quad T_{4}=T_{5}, \quad T_{9}=-T_{10}, \quad T_{12}=0, \quad T_{14}=T_{15},
$$$$
T_{19}=-T_{20}, \quad T_{23}=T_{24}, \quad T_{29}=T_{30} .
$$

\section{Appendix B: Invariant amplitudes}

Here we provide expressions for the linear combinations of invariant amplitudes $\bar{A}_{i}\left(Q^{2}\right)=\bar{A}_{i}\left(0,-Q^{2},-Q^{2}\right)$ that contribute to the generalized polarizabilities, see Eqs. (9a)-(9f):

$$
\begin{array}{r}
g_{1}=\bar{A}_{1}, \quad g_{2}=\bar{A}_{2}, \quad g_{3}=\bar{A}_{5}, \quad g_{4}=\bar{A}_{5}+\bar{A}_{7}+4 \bar{A}_{11}, \\
g_{5}=2 \bar{A}_{6}+\bar{A}_{9}, \quad g_{6}=\bar{A}_{12} .
\end{array}
$$

The results are given for the $\pi \mathrm{N}$ loop and Delta pole contributions; for the $\pi \Delta$ loop results, see supplementary material to this article.

\section{1. $\pi N$ loops}

Here, $D_{1}(x, y)=\left[\tau(1-x)^{2}\left(1-4 y^{2}\right)+D_{N}(x)\right]^{-1}$, $D_{2}(x, y)=\left[\tau x^{2}\left(1-4 y^{2}\right)+D_{N}(x)\right]^{-1}, \quad D_{0}(x, y)$ $=\left[D_{N}(x)\right]^{-1}$, and $D_{\pi}(x, y)=\left[4 \tau(1-x) x+\mu^{2}\right]^{-1}$. In turn, $D_{N}(x)=x^{2}+\mu^{2}(1-x)$. The amplitudes are expressed as integrals over the Feynman parameters as follows:

$g_{i}\left(Q^{2}\right)=\frac{g_{A}^{2}}{8 \pi^{2} f_{\pi}^{2} M^{n_{i}}} \int_{0}^{1} \mathrm{~d} x \int_{-1 / 2}^{1 / 2} \mathrm{~d} y \phi_{i}(\tau, x, y)$,

where $\phi_{i}$ are given below, $g_{A}=1.27$ and $f_{\pi}=92.21 \mathrm{MeV}$ are the axial coupling constant and the pion decay constant, and $n_{i}=1,3,2,2,3,3$ for $i=1,2,3,4,5,6$ account for the correct dimensions of the respective $g_{i}$.

\section{a. Proton}

$$
\begin{aligned}
\phi_{1}= & -\frac{1}{2} D_{1}^{2}(x, y)(x-1)^{3} x\left(-4 y^{2}+2 x+1\right) \\
& -\frac{1}{4} D_{2}^{2}(x, y) x^{4}\left(4 y^{2}+2 x-1\right) \\
& -\frac{1}{2} D_{0}^{2}(x, y) x^{3}\left(3 x^{2}-5 x+2\right)-2 D_{\pi}(x, y)(x-1)^{2} x, \quad(\mathrm{~B} 3) \\
\phi_{2}= & 4 D_{1}^{4}(x, y) y^{2}\left(4 y^{2}-1\right) \tau(x-1)^{6} x^{3} \\
& -\frac{1}{3} D_{1}^{3}(x, y)(x-1)^{4} x\left(4 y^{2}\left(4 y^{2}-1\right) \tau\right. \\
& \left.+x^{2}\left(8 y^{2} \tau-1\right)-4 y^{2}\left(4 y^{2}+1\right) \tau x\right) \\
& +\frac{1}{24} D_{1}^{2}(x, y)(x-1)^{3} x\left(\left(36 y^{2}-7\right) x+4\right) \\
& +2 D_{2}^{4}(x, y) y^{2}\left(4 y^{2}-1\right) \tau(x-1)^{2} x^{7} \\
& -\frac{1}{12} D_{2}^{3}(x, y)(x-1) x^{5}\left(\tau\left(-48 y^{4}+2\left(36 y^{4}-5 y^{2}+1\right) x-1\right)\right. \\
& \left.+2\left(3 y^{2}(x-2)-1\right)\right)
\end{aligned}
$$




$$
\begin{aligned}
& +\frac{1}{16} D_{2}^{2}(x, y) x^{3}\left(-4 y^{2}+\left(4 y^{2}+5\right) x^{2}-8 x+3\right) \\
& -\frac{3}{2} D_{0}^{4}(x, y)(x-1)^{2} x^{4}\left(\mu^{2}+\left(\mu^{2}-1\right) x^{2}-2 \mu^{2} x\right) \\
& +\frac{1}{12} D_{0}^{3}(x, y)(x-1) x^{2}\left(-18 \mu^{2}+10 x^{4}+9\left(\mu^{2}-3\right) x^{3}\right. \\
& \left.+\left(26-36 \mu^{2}\right) x^{2}+45 \mu^{2} x\right) \\
& -\frac{1}{6} D_{0}^{2}(x, y)(x-2)(x-1)^{2} \text {, } \\
& \phi_{3}=\frac{1}{2} D_{1}^{2}(x, y)(x-1)^{2} x\left(8 y^{2}+\left(8 y^{2}+1\right) x^{2}-16 y^{2} x\right) \\
& +\frac{1}{4} D_{2}^{2}(x, y) x^{4}\left(8 y^{2} x+x-1\right) \\
& +\frac{1}{4} D_{0}^{2}(x, y) x^{3}\left(3 x^{2}-5 x+2\right), \\
& \phi_{4}=\frac{1}{2} D_{1}^{2}(x, y)(x-1)^{3} x\left(4 y^{2}(4 x-3)+1\right) \\
& +\frac{1}{4} D_{2}^{2}(x, y) x^{4}\left(4 y^{2}(4 x-3)-1\right), \\
& \times\left(4 y^{2}\left(\tau\left(2 x^{2}+x-8\right)+3(x-2) x\right)\right. \\
& \left.-48 y^{4} \tau(x-2) x+x(\tau(x+1)+x-3)-2\right) \\
& +\frac{1}{24} D_{2}^{2}(x, y)(x-1) x^{2}\left(4 x^{2}+24 y^{2}(2 x-1) x+x+1\right) \text {, } \\
& \phi_{3}=-\frac{1}{2} D_{1}^{2}(x, y)(x-1) x\left(8 y^{2}+\left(8 y^{2}+1\right) x^{2}-16 y^{2} x\right) \\
& +\frac{1}{2} D_{2}^{2}(x, y) x^{3}\left(8 y^{2} x+x-1\right), \\
& \phi_{4}=\frac{1}{2} D_{1}^{2}(x, y)(x-1)^{3} x\left(4 y^{2}(4 x-3)+1\right) \\
& +\frac{1}{4} D_{2}^{2}(x, y) x^{4}\left(4 y^{2}(4 x-3)-1\right), \\
& \phi_{5}=-\frac{1}{6} D_{1}^{3}(x, y)(x-1)^{2} x^{3}\left(\left(4 y^{2}-1\right) \tau(x-1)^{2}-(x-2) x\right) \\
& -\frac{1}{12} D_{1}^{2}(x, y)(x-1)^{2} x\left(12 y^{2}+4 x^{2}-3\left(4 y^{2}+1\right) x-3\right) \\
& -\frac{1}{12} D_{2}^{3}(x, y)(x-1) x^{5}\left(( 4 y ^ { 2 } - 1 ) \tau \left(-20 y^{2}+12 y^{2} x\right.\right. \\
& \left.+x+1) s+36 y^{2}-12 y^{2} x-x-1\right) \\
& -\frac{1}{24} D_{2}^{2}(x, y)(x-1) x^{3}\left(-36 y^{2}+\left(48 y^{2}+4\right) x+3\right) \text {, } \\
& \times x\left(-4 y^{2}+\left(12 y^{2}-1\right) x+1\right) \\
& +\frac{1}{12} D_{2}^{3}(x, y)(x-1) x^{5}\left(-12 y^{2}+\left(4 y^{2}-1\right)\right. \\
& \left.\times \tau\left(-8 y^{2}+2\left(6 y^{2}-1\right) x+1\right)+2 x-1\right) \\
& -\frac{1}{48} D_{2}^{2}(x, y) x^{3}\left(-12 y^{2}+16 x^{2}+\left(12 y^{2}-25\right) x+9\right) \\
& +\frac{3}{2} D_{0}^{4}(x, y)(x-1)^{2} x^{4}\left(\mu^{2}+\left(\mu^{2}-1\right) x^{2}-2 \mu^{2} x\right) \\
& -\frac{1}{12} D_{0}^{3}(x, y)(x-1) x^{2}\left(-18 \mu^{2}+10 x^{4}+9\left(\mu^{2}-3\right) x^{3}\right. \\
& \left.+\left(26-36 \mu^{2}\right) x^{2}+45 \mu^{2} x\right) \\
& +\frac{1}{12} D_{0}^{2}(x, y)(x-1)^{2} x^{2}(5 x-6), \\
& \phi_{6}=-4 D_{1}^{3}(x, y) y^{2}(x-1)^{4} x^{3}-2 D_{2}^{3}(x, y) y^{2}(x-1) x^{6} \text {. } \\
& \phi_{1}=\frac{1}{2} D_{1}^{2}(x, y)(x-1)^{2} x\left(-4 y^{2}+4 y^{2} x+x+1\right) \\
& -\frac{1}{2} D_{2}^{2}(x, y)\left(4 y^{2}+1\right) x^{4} \\
& -2 D_{\pi}(x, y)(x-1)^{2} x, \\
& \phi_{2}=-4 D_{1}^{4}(x, y) y^{2}\left(4 y^{2}-1\right) \tau(x-1)^{5} x^{3} \\
& +\frac{1}{12} D_{1}^{3}(x, y)(x-1)^{2} x \\
& \times\left(4 y^{2} \tau\left(x^{2}+x-2\right)^{2}-x^{2}\left(\tau(x-1)^{2}\right.\right. \\
& \left.+x(x+2)-4)+64 y^{4} \tau(x-1)^{3}\right) \\
& +\frac{1}{24} D_{1}^{2}(x, y)(x-1)^{2} x(x(4 x-1)-4) \\
& +4 D_{2}^{4}(x, y) y^{2}\left(4 y^{2}-1\right) \tau(x-1)^{2} x^{6} \\
& -\frac{1}{12} D_{2}^{3}(x, y)(x-1) x^{4} \\
& g_{1}=\frac{f_{M}\left[2 g_{M} M_{+}\left(4 M^{2}+Q^{2}\right)-g_{E} Q^{2} \Delta\right]+g_{E} Q^{2}\left(g_{M} M_{+}-2 g_{E} \Delta\right)}{4 M^{2} M_{+}^{3} \Delta}, \\
& g_{2}=-\frac{f_{M} g_{M} M_{+}-g_{E}^{2} \Delta}{2 M^{2} M_{+}^{3} \Delta} \\
& g_{3}=\frac{f_{M}\left(8 M^{2}+Q^{2}\right)\left(g_{M} M_{+}-2 g_{E} \Delta\right)+g_{E}\left[2 g_{M} M_{+} Q^{2}-g_{E} \Delta\left(8 M^{2}+Q^{2}\right)\right]}{8 M^{3} M_{+}^{3} \Delta}, \\
& g_{4}=\frac{f_{M} g_{M} M_{+}-2 f_{M} g_{E} \Delta-2 g_{E}^{2} \Delta+3 g_{E} g_{M} M_{+}}{M M_{+}^{3} \Delta}, \\
& g_{5}=-\frac{f_{M} g_{M} M_{+}-3 f_{M} g_{E} \Delta-g_{E}^{2} \Delta+3 g_{E} g_{M} M_{+}}{4 M^{2} M_{+}^{3} \Delta}, \\
& g_{6}=-\frac{f_{M} g_{M} M_{+}-2 f_{M} g_{E} \Delta-g_{E}^{2} \Delta+2 g_{E} g_{M} M_{+}}{4 M^{2} M_{+}^{3} \Delta} .
\end{aligned}
$$

\section{b. Neutron}




\section{References}

1. R.A. Berg, C.N. Lindner, Phys. Rev. 112, 2072 (1958)

2. R.A. Berg, C.N. Lindner, Nucl. Phys. 26, 259 (1961)

3. P.A.M. Guichon, G.Q. Liu, A.W. Thomas, Nucl. Phys. A 591, 606 (1995). arXiv:nucl-th/9605031

4. P.A.M. Guichon, M. Vanderhaeghen, Prog. Part. Nucl. Phys. 41, 125 (1998). arXiv:hep-ph/9806305

5. M. Gorchtein, C. Lorcé, B. Pasquini, M. Vanderhaeghen, Phys. Rev. Lett. 104, 112001 (2010)

6. D. Drechsel, G. Knöchlein, A.Y. Korchin, A. Metz, S. Scherer, Phys. Rev. C 57, 941 (1998)

7. D. Drechsel, G. Knöchlein, A.Y. Korchin, A. Metz, S. Scherer, Phys. Rev. C 58, 1751 (1998)

8. P. Bourgeois et al., Phys. Rev. Lett. 97, 212001 (2006). arXiv:nucl-ex/0605009

9. P. Bourgeois et al., Phys. Rev. C 84, 035206 (2011)

10. J. Roche et al. (VCS and A1 Collaborations), Phys. Rev. Lett. 85, 708 (2000). arXiv:hep-ex/0007053

11. P. Janssens et al. (A1 Collaboration), Eur. Phys. J. A 37, 1 (2008). arXiv:0803.0911 [nucl-ex]

12. N. d'Hose, Eur. Phys. J. A 28S1, 117 (2006)

13. L. Doria et al. (A1 Collaboration), Phys. Rev. C 92, 054307 (2015). arXiv:1505.06106 [nucl-ex]

14. L. Correa, Measurement of the generalized polarizabilities of the proton by virtual Compton scattering at MAMI and $Q^{2}=$ $0.2 \mathrm{GeV}^{2}$, Ph.D. Thesis, Mainz-Clermont-Ferrand (2016)

15. T.R. Hemmert, B.R. Holstein, G. Knöchlein, S. Scherer, Phys. Rev. Lett. 79, 22 (1997). arXiv:nucl-th/9705025

16. T.R. Hemmert, B.R. Holstein, G. Knöchlein, S. Scherer, Phys. Rev. D 55, 2630 (1997). arXiv:nucl-th/9608042

17. T.R. Hemmert, B.R. Holstein, G. Knöchlein, D. Drechsel, Phys. Rev. D 62, 014013 (2000)

18. C.W. Kao, M. Vanderhaeghen, Phys. Rev. Lett. 89, 272002 (2002). arXiv:hep-ph/0209336

19. C.W. Kao, B. Pasquini, M. Vanderhaeghen, Phys. Rev. D 70, 114004 (2004). arXiv:hep-ph/0408095 [Erratum: Phys. Rev. D 92, 119906 (2015)]

20. B. Pasquini, M. Gorchtein, D. Drechsel, A. Metz, M. Vanderhaeghen, Eur. Phys. J. A 11, 185 (2001). arXiv:hep-ph/0102335

21. D. Drechsel, B. Pasquini, M. Vanderhaeghen, Phys. Rep. 378, 99 (2003). arXiv:hep-ph/0212124

22. T. Becher, H. Leutwyler, Eur. Phys. J. C 9, 643 (1999). arXiv:hep-ph/9901384

23. T. Fuchs, J. Gegelia, G. Japaridze, S. Scherer, Phys. Rev. D 68, 056005 (2003). arXiv:hep-ph/0302117

24. V. Pascalutsa, B.R. Holstein, M. Vanderhaeghen, Phys. Lett. B 600, 239 (2004). arXiv:hep-ph/0407313

25. B.R. Holstein, V. Pascalutsa, M. Vanderhaeghen, Phys. Rev. D 72, 094014 (2005). arXiv:hep-ph/0507016

26. V. Pascalutsa, M. Vanderhaeghen, Phys. Rev. D 73, 034003 (2006). arXiv:hep-ph/0512244

27. T. Ledwig, J. Martin-Camalich, V. Pascalutsa, M. Vanderhaeghen, Phys. Rev. D 85, 034013 (2012). arXiv:1108.2523 [hep-ph]

28. J.M. Alarcón, J. Martin-Camalich, J.A. Oller, Phys. Rev. D 85, 051503 (2012). arXiv:1110.3797 [hep-ph]

29. J.M. Alarcón, J. Martin-Camalich, J.A. Oller, Ann. Phys. 336, 413 (2013). arXiv:1210.4450 [hep-ph]

30. A.N. Hiller Blin, T. Ledwig, M.J. Vicente Vacas, Phys. Rev. D 93, 094018 (2016). arXiv:1602.08967 [hep-ph]

31. D.L. Yao, D. Siemens, V. Bernard, E. Epelbaum, A.M. Gasparyan, J. Gegelia, H. Krebs, U.-G. Meißner, JHEP 1605, 038 (2016). arXiv:1603.03638 [hep-ph]

32. V. Pascalutsa, Prog. Part. Nucl. Phys. 55, 23 (2005). arXiv:nucl-th/0412008
33. J.M.M. Hall, V. Pascalutsa, Eur. Phys. J. C 72, 2206 (2012). arXiv:1203.0724 [hep-ph]

34. V. Bernard, E. Epelbaum, H. Krebs, U.G. Meißner, Phys. Rev. D 87, 054032 (2013). arXiv:1209.2523 [hep-ph]

35. V. Lensky, V. Pascalutsa, Eur. Phys. J. C 65, 195 (2010). arXiv:0907.0451 [hep-ph]

36. V. Lensky, J.M. Alarcón, V. Pascalutsa, Phys. Rev. C 90, 055202 (2014). arXiv:1407.2574 [hep-ph]

37. V. Lensky, J.A. McGovern, V. Pascalutsa, Eur. Phys. J. C 75, 604 (2015). arXiv:1510.02794 [hep-ph]

38. G. Eichmann, C.S. Fischer, Phys. Rev. D 87, 036006 (2013). arXiv:1212.1761 [hep-ph]

39. F.E. Low, Phys. Rev. 96, 1428 (1954)

40. S. Scherer, A.Y. Korchin, J.H. Koch, Phys. Rev. C 54, 904 (1996). arXiv:nucl-th/9605030

41. V. Pascalutsa, M. Vanderhaeghen, Phys. Rev. D 91, 051503 (2015). arXiv: 1409.5236 [nucl-th]

42. V. Lensky, V. Pascalutsa, M. Vanderhaeghen, C.W. Kao. arXiv:1701.01947 [hep-ph]

43. F. Hagelstein, R. Miskimen, V. Pascalutsa, Prog. Part. Nucl. Phys. 88, 29 (2016). arXiv:1512.03765 [nucl-th]

44. M. Vanderhaeghen, Phys. Lett. B 402, 243 (1997)

45. S. Weinberg, Physica A 96, 327 (1979)

46. J. Gasser, H. Leutwyler, Ann. Phys. 158, 142 (1984)

47. J. Gasser, M.E. Sainio, A. Švarc, Nucl. Phys. B 307, 779 (1988)

48. V. Pascalutsa, M. Vanderhaeghen, S.N. Yang, Phys. Rep. 437, 125 (2007)

49. L. Geng, Front. Phys. (Beijing) 8, 328 (2013). arXiv:1301.6815 [nucl-th]

50. V. Pascalutsa, D.R. Phillips, Phys. Rev. C 67, 055202 (2003). arXiv:nucl-th/0212024

51. R. Tarrach, Nuovo Cim. A 28, 409 (1975)

52. V. Lensky, J.A. McGovern, Phys. Rev. C 89, 032202 (2014). arXiv:1401.3320 [nucl-th]

53. A. Metz, D. Drechsel, Z. Phys. A 356, 351 (1996)

54. A. Metz, D. Drechsel, Z. Phys. A 359, 165 (1997). arXiv:nucl-th/9705010

55. T.R. Hemmert, B.R. Holstein, J. Kambor, Phys. Lett. B 395, 89 (1997). arXiv:hep-ph/9606456

56. D. Drechsel, S.S. Kamalov, L. Tiator, Eur. Phys. J. A 34, 69 (2007). arXiv:0710.0306 [nucl-th]

57. H.W. Grießhammer, J.A. McGovern, D.R. Phillips, G. Feldman, Prog. Part. Nucl. Phys. 67, 841 (2012). arXiv:1203.6834 [nucl-th]

58. B. Pasquini, D. Drechsel, M. Vanderhaeghen, Phys. Rev. C 76, 015203 (2007). arXiv:0705.0282 [hep-ph]

59. J.A. McGovern, D.R. Phillips, H.W. Grießhammer, Eur. Phys. J. A 49, 12 (2013). arXiv:1210.4104 [nucl-th]

60. H.W. Grießhammer, J.A. McGovern, D.R. Phillips, Eur. Phys. J. A 52, 139 (2016). arXiv:1511.01952 [nucl-th]

61. P.P. Martel et al. (A2 Collaboration), Phys. Rev. Lett. 114, 112501 (2015). arXiv:1408.1576 [nucl-ex]

62. K.A. Olive et al. (Particle Data Group Collaboration), Chin. Phys. C 38, 090001 (2014)

63. V. Olmos de León et al., Eur. Phys. J. A 10, 207 (2001)

64. R. Bradford, A. Bodek, H.S. Budd, J. Arrington, Nucl. Phys. Proc. Suppl. 159, 127 (2006). arXiv:hep-ex/0602017

65. Jefferson Lab Experiment C12-15-001, Co-spokespersons $\mathrm{M}$. Paolone, N. Sparveris (contact person), A. Camsonne, M. Jones, PAC44. https://www.jlab.org/exp_prog/proposals/16/C12-15-001

66. J.A.M. Vermaseren, arXiv:math-ph/0010025

67. D. Binosi, J. Collins, C. Kaufhold, L. Theussl, Comput. Phys. Commun. 180, 1709 (2009). arXiv:0811.4113 [hep-ph] 